\title{
Disrupting D2-NMDA receptor heteromerization blocks the rewarding effects of cocaine but preserves natural reward processing
}

Andry Andrianarivelo ${ }^{1-3}$, Estefani Saint-Jour ${ }^{1-3}$, Paula Pousinha ${ }^{4,5}$, Sebastian P. Fernandez ${ }^{4,5}$, Anna Petitbon $^{6}$, Veronique De Smedt-Peyrusse ${ }^{6}$, Nicolas Heck ${ }^{1-3}$, Vanesa Ortiz ${ }^{4,5}$, Marie-Charlotte Allichon ${ }^{1-3}$, Vincent Kappès ${ }^{1-3}$, Sandrine Betuing ${ }^{1-3}$, Roman Walle ${ }^{6}$, Ying Zhu ${ }^{7,8}$, Charlène Joséphine ${ }^{9}$, Alexis-Pierre Bemelmans ${ }^{9}$, Gustavo Turecki ${ }^{10}$, Naguib Mechawar ${ }^{10}$, Jonathan A Javitch ${ }^{7,8,11}$, Jocelyne Caboche ${ }^{1-3}$, Pierre Trifilieff $^{6}$, Jacques Barik ${ }^{4,5}$, Peter Vanhoutte*1-3

${ }^{1}$ CNRS, UMR 8246, Neuroscience Paris Seine, F-75005, Paris, France

${ }^{2}$ INSERM, UMR-S 1130, Neuroscience Paris Seine, Institute of Biology Paris Seine, F-75005, Paris, France

${ }^{3}$ Sorbonne Université, UPMC Université Paris 06, UM CR18, Neuroscience Paris Seine, F-75005, Paris, France

${ }^{4}$ Université Côte d'Azur, Nice, France

${ }^{5}$ Institut de Pharmacologie Moléculaire \& Cellulaire, CNRS UMR7275, Valbonne, France

${ }^{6}$ Université Bordeaux, INRAE, Bordeaux INP, NutriNeuro, 33000, Bordeaux, France

${ }^{7}$ Division of Molecular Therapeutics, New York State Psychiatric Institute, New York, NY 10032, USA.

${ }^{8}$ Department of Psychiatry, Columbia University, New York, NY 10032, USA.

${ }^{9}$ Commissariat à l'Énergie Atomique et aux Énergies Alternatives (CEA), Département de la Recherche Fondamentale, Institut de biologie François Jacob, MIRCen, and CNRS UMR 9199, Université Paris-Sud, Université Paris-Saclay, Neurodegenerative Diseases Laboratory, Fontenay-aux-Roses, France.

${ }^{10}$ Douglas MentalHealth University Institute, Department of Psychiatry, McGill University, Montreal, QC, Canada.

${ }^{11}$ Department of Pharmacology, Columbia University, New York, NY 10032, USA.

*Corresponding author: peter.vanhoutte@upmc.fr 


\begin{abstract}
Addictive drugsincrease dopamine in the nucleus accumbens (NAc), where it persistently shapes excitatory glutamate transmission and hijacks natural reward processing. Herein, we provide evidence, from mice to human, that an underlying mechanism relies on drug-evoked heteromerization of glutamate NMDA receptors (NMDAR) with dopamine receptor 1 (D1R) or 2 (D2R). Using temporally-controlled inhibition of D1R-NMDAR heteromerization, we unraveled their selective implication in early developmental phases of cocaine-mediated synaptic, morphological and behavioral responses. In contrast, preventing D2RNMDAR heteromerization blocked the persistence of these adaptations. Importantly, interfering with these heteromers spared natural reward processing. Strikingly, we established that D2R-NMDAR complexes exist in human samples and showed that, despite a decreased D2R protein expression in the NAc, psychostimulant-addicts display a higher proportion of $D 2 R$ forming heteromers with NMDAR. These findings contribute to a better understanding of molecular mechanisms underlying addiction and uncover D2R-NMDAR heteromers as targets with potential therapeutic value.
\end{abstract}




\section{Introduction}

Drug addiction is characterized by compulsive patterns of drug-seeking and drug-taking behavior in spite of detrimental consequences and a high rate of relapse after withdrawal. A hallmark of addictive drugs is their ability to increase dopamine concentration in discrete brain regions, which persistently shapes excitatory glutamate transmission within the reward circuit, thereby hijacking natural reward processing $(1$, 2). This calls for a better understanding of the precise molecular events underlying the detrimental interplay between dopamine and glutamate signaling triggered by drugs of abuse.

The enduring behavioral alterations induced by protracted drug exposure are largely believed to result from persistent drug-evoked neuronal adaptations within the striatum, especially in its ventral part, the nucleus accumbens (NAc) $(1,3)$. The striatum is indeed a key target structure of drugs of abuse that integrates convergent glutamate inputs from limbic, thalamic and cortical regions, encoding components of drugassociated stimuli and environment, and dopamine signals that mediate reward prediction error and incentive values (4). Integration of dopamine and glutamate signals is achieved by the two segregated subpopulations of GABAergic medium-sized spiny neurons (MSN) expressing either the dopamine receptor (DAR) type 1 (D1R) or type 2 (D2R), although a fraction of MSN in the NAc expresses both receptors (5). Cell-type-specific manipulations of neuronal activity showed that inhibiting and activating D1R-MSN respectively dampens and potentiates long-term drug-evoked responses, in line with their "proreward" action (2, 6-9). By contrast, the majority of studies supports an inhibitory role of D2R-MSN activation on drug-mediated adaptations $(6,7,10-12)$. These studies, based on direct manipulations of MSN activity, were extremely instrumental to highlight the role of MSNs as putative players in drug-related behavioral adaptations. However, they do not establish how drugs of abuse persistently impact the functionality of each MSN subpopulation or the underlying cellular and molecular mechanisms. In this context, increasing evidence suggests that such a central role of MSN subpopulations in drug-induced behavioral adaptations originates, at least in part, from dopamine-dependent long-lasting changes at excitatory striatal synapses. Indeed, long-term potentiation of specific glutamatergic afferences impinging onto D1R-MSN induced by dopamine is responsible for both the induction and maintenance of long-lasting behavioral adaptations to repeated cocaine exposure (13-15). Interestingly, glutamate transmission onto D2R-MSN seems to be spared by cocaine exposure, but selectively altered during cocaine craving after long access to high doses of cocaine (16).

It is therefore timely to identify molecular mechanisms by which drug-evoked increases in dopamine can permanently hijack glutamate transmission onto MSN. Although a number of studies have described the crosstalk between D1R and glutamate receptor of the NMDA (NMDAR) subtype as a key player in the behavioral effects of psychostimulants $(2,17-21,21,22)$, the underlying molecular mechanisms remain 
elusive. Moreover, the processes by which dopamine impairs D2R-MSN activity to promote long-lasting drug-induced reinforcement is yet unknown.

Heteromeric complexes formed between dopamine receptors (DAR) and glutamate NMDAR have been proposed as integrators of dopamine and glutamate signals in both MSN populations (23). Receptor heteromers are of particular interest, not only because of their ability to dynamically modulate the component receptor's functions in time and space, but also because they exhibit functional properties distinct from the component receptors, making them attractive targets for the development of more selective pharmacological strategies (24-27). Most evidence generated to date regarding dopamine-NMDA receptor heteromer functions come from in vitro and ex vivo studies and their potential role in long-term druginduced adaptations has been overlooked. D1R form heteromers with GluN1 subunits of NMDAR in vitro $(28,29)$ and in vivo in the striatum $(30,31)$ and have been shown to allow the facilitation of NMDAR signaling by dopamine in D1R-MSN ex vitro on striatal slices (31). By contrast, the binding of D2R to GluN2B subunits of NMDAR mediates the inhibition of NMDA currents by dopamine in D2R-MSN and controls acute stereotypic locomotor responses to high cocaine doses (32). DAR-NMDAR heteromers therefore appear as putative molecular platforms that mediate crosstalk between dopamine and glutamate transmission onto MSNs. However, whether such receptor heteromers might constitute molecular substrates by which drugs of abuse enduringly alter glutamate transmission and trigger long-lasting behavioral alterations has not been studied.

We therefore investigated D1R-GluN1 and D2R-GluN2B heteromerization in the striatum in vivo in response to repeated cocaine exposure. We found that cocaine triggers a transient increase of D1R-GluN1 heteromerization in the entire striatum, which returns to baseline level upon withdrawal from the drug. By contrast, cocaine induces a stable heteromerization of D2R-Glu2NB that is mostly restricted to the NAc and persists over a withdrawal period. Using a temporally-controlled disruption these receptor heteromers, combined with electrophysiological recordings, imaging and behavioral assessments, we showed that D1RGluN1 heteromerization controls the development of cocaine-evoked long-term synaptic plasticity and morphological changes in D1R-MSNs, as well as behavioral adaptations. In contrast, D2R-GluN2B heteromerization mediates the persistence of these adaptations after a withdrawal period followed by a reexposure to the drug. Importantly, the targeting of either type of heteromers preserves natural reward processing. Strikingly, we found that such receptor complexes also exist in human post-mortem brain samples and showed that, despite a substantial decrease of D2R protein expression, psychostimulant-addicts display a significantly higher proportion of D2R that form heteromers with GluN2B in the NAc. Our results support a model by which the heteromerization of dopamine and glutamate receptors induced by drugs of abuse in D1R- and D2R-MSNs is a key endogenous molecular event underlying the detrimental interplay 
between these two neurotransmitter systems in drug addiction. The role of D2R-GluN2B heteromers in the persistence of the sensitizing and rewarding effects of drugs makes them potential targets not only for addiction in humans, but also more broadly in multiple neuropsychiatric disorders.

\section{Results}

Behavioral sensitization to cocaine is associated with transient D1R-NMDAR heteromerization and prolonged D2R-GluN2B heteromerization in the NAc

Before studying the role of DAR-NMDAR heteromers in cocaine-evoked adaptations, we investigated whether a cocaine regimen that triggers persistent behavioral adaptations can modulate the formation of these receptor complexes in vivo in the striatum. Mice were subjected to five daily injections of cocaine (15 $\mathrm{mg} / \mathrm{kg}$ ), which elicits a progressive locomotor sensitization (Fig. 1A) that is known to persist for several weeks after cocaine withdrawal. This behavioral paradigm is a straightforward model to study the mechanisms involved in drug-induced behavioral adaptations $(2,33)$. Mice were sacrificed one day after the last injection to detect endogenous DAR-NMDAR proximity in distinct striatal sub-regions through Proximity Ligation Assay (PLA) (34). The brightfield PLA assay yielded a brown punctate signal for D1RGluN1 and D2R-GluN2B complexes that was absent when one of the two primary antibodies was omitted (Fig. 1B), similar to what was previously found when PLA was performed in DA receptor KO mice (31, 34, 35). Using this approach, we found that cocaine-treated mice displayed increased D1R-GluN1 heteromerization in the dorso-lateral (DL Str) and dorso-medial (DM Str) striatum, as well as in the nucleus accumbens core (NAc core) and shell (NAc shell) sub-divisions (Fig. 1C). Cocaine also increased D2RGluN2B heteromerization, but primarily in the NAc, with a smaller effect in the DL Str (Fig. 1D). This increased heteromerization occurred in the absence of changes in global expression levels of the component receptors (Fig. S1A,B). Of note, and as previously observed (36), repeated cocaine exposure decreases expression levels of the synaptic scaffold protein PSD-95 (Fig. S1C), which could partly explain our results as the interaction of NMDAR and D1R with PSD-95, through partly overlapping domains, has been described to prevent D1R-GluN1 interaction (37).

To study the kinetics of receptor heteromerization, mice were subjected to a cocaine-induced locomotor sensitization paradigm followed by one-week withdrawal and a challenge injection of saline or cocaine. We found that cocaine-mediated D1R-GluN1 heteromerization returned to baseline levels upon withdrawal but increased again, in all striatal sub-regions except in the DL-Str, after the challenge injection of cocaine (Fig. 1E). 
bioRxiv preprint doi: https://doi.org/10.1101/2021.01.25.428078; this version posted January 26, 2021. The copyright holder for this preprint (which was not certified by peer review) is the author/funder, who has granted bioRxiv a license to display the preprint in perpetuity. It is made available under aCC-BY-NC-ND 4.0 International license.

A

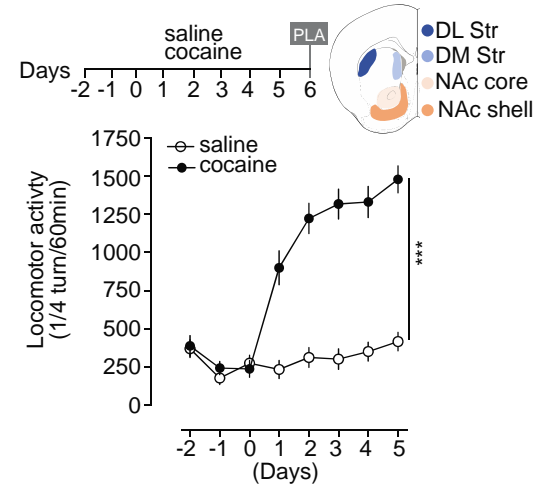

C

10
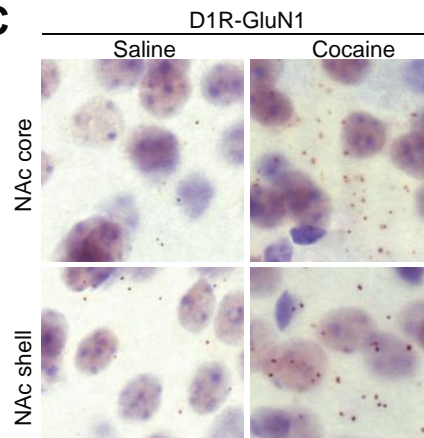

$\sum_{\Delta}^{\infty}$

के

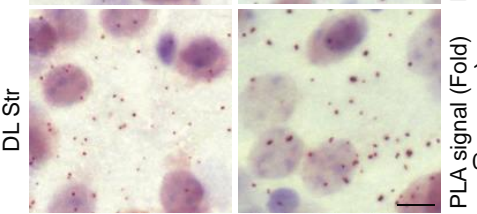

E
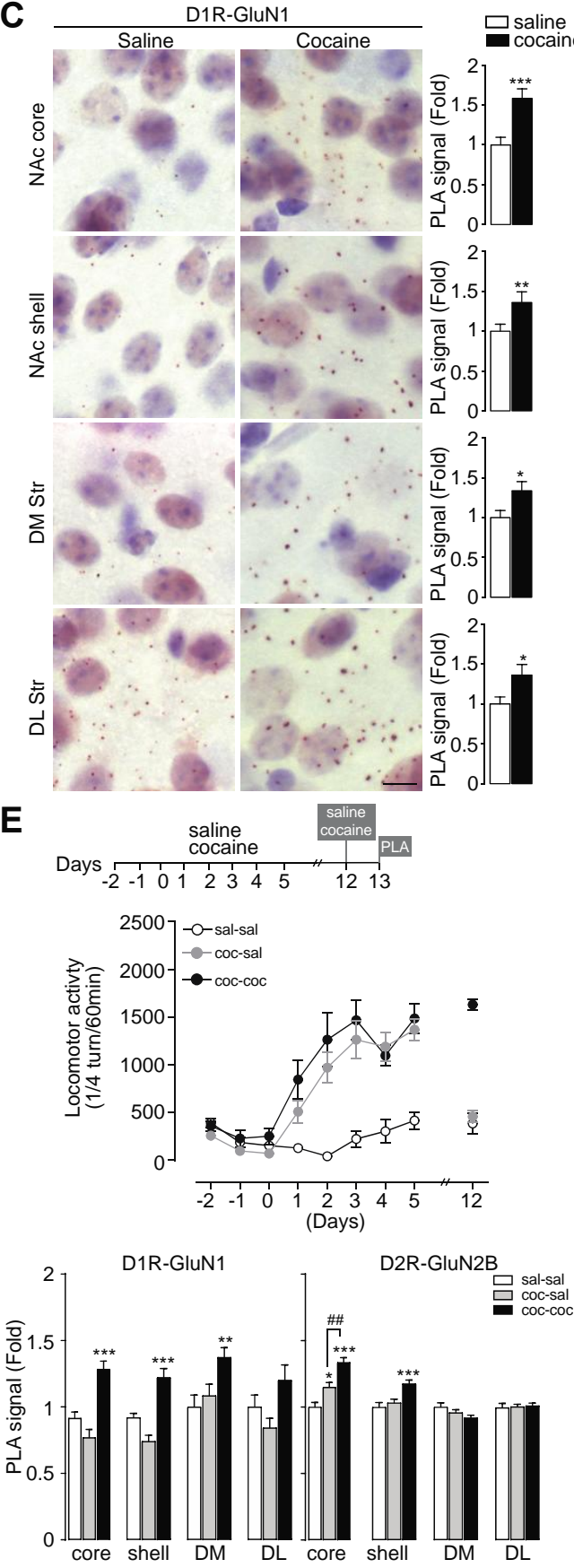

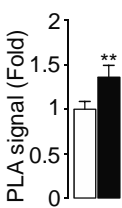
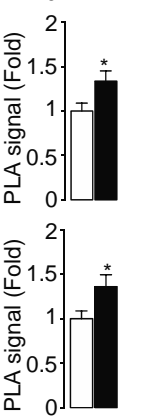

\section{s}

B 
Figure 1. Behavioral sensitization to cocaine is associated with transient D1R-NMDAR heteromerization and prolonged D2R-GluN2B heteromerization in the NAc. (A) Experimental time frame (DL Str: dorso-lateral striatum; DMStr dorso-medial striatum; nucleus accumbens core (NAc core) and shell (NAc shell) and measurements of locomotoractivity prior and during 5 days of saline or cocaine $(15 \mathrm{mg} / \mathrm{kg})$ injections. Two -way ANOVA, treatment effect, $\mathrm{F}(1,24)=67.79$, $* * * \mathrm{P}<0.0001$ saline v s cocaine on day $5, \mathrm{n}=13$ mice/group. (B) Example images of D1RGluN1 and D2R-GluN2B heteromer PLA detection and related negative (Neg. Cont) showing the absence of signal when one primary antibody is omitted. (C) Detection and quantifications of D1R-GluN1 heteromerization in salineand cocaine-treated groups. PLA signal is represented as fold increase normalized to the saline group. Two-sided Student's $t$-test. $* P<0.05$; ** $P<0.01$; *** $P<0.001$ saline vs cocaine, $\mathrm{n}=28-84$ fields of view/structure (NAc core: 4, NAc shell: 12; DM: 6, DL: 6, fields of view/mice) from 7 mice/group. (D) Same as for (C) for D2R-GluN2B heteromerization. (E) Experimental time frame, measurements of locomotor activity and quantifications of D1RGluN1 and D2R-GluN2Bheteromerization.PLA signal is represented as fold increase normalized to the saline group. One-way ANOVA. $* P<0.05 ; * * P<0.01 ; * * * P<0.001$ saline-saline vs cocaine-saline/cocaine-cocaine, \#\# $\mathrm{p}<0.01$ cocaine-saline vs cocaine-cocaine, $n=28-84$ fields of view/structure from 7 mice/group. (F) Same as for (E) except that mice received either a vehicle solution (veh) or the D1R antagonist SCH23390 (SCH) or the D2R antagonist Eticlopride (Etic) prior to a cocaine challenge. One-way ANOVA. * $P<0.05 ; * * P<0.01 ; * * * P<0.001$ vehiclesaline vs vehicle-cocaine, \# p < 0.05, \#\# p<0.01 vehicle-cocaine vs SCH-cocaine or cocaine vs Etic-cocaine, n.s: not significant, $\mathrm{n}=28-84$ fields of view/structure from 7 mice/group. (B-D) scale bar: $10 \mu \mathrm{m}$. Error bars denote s.e.m.

Strikingly, D2R-GluN2B heteromerization appeared to be sustained over the withd rawal period specifically in the NAc core. The challenge injection of cocaine further increased D2R-Glu2NB heteromers in the NAc, but not in dorsal parts of the striatum (Fig. 1E). To assess the role of D1R or D2R stimulation for receptor heteromerization, mice underwent a cocaine-locomotor sensitization followed by a withdrawal. Before a cocaine challenge, mice received an intraperitoneal injection of D1R or D2R antagonist that blunted the expression of behavioral sensitization. This allowed us to show that the stimulation of D1R or D2R was mandatory for cocaine-induced D1R-GluN1 and D2R-GluN2B heteromerization, respectively (Fig. 1F). Altogether, these data show that behavioral sensitization to cocaine is associated with a dopamine receptordependent transient heteromerization of D1R-GluN1 in the whole striatum, whereas D2R-GluN2B heteromerization occurs primarily in the NAc and is maintained during cocaine withdrawal in the core subdivision.

\section{Cocaine-evoked potentiation of glutamate transmission onto D1R-MSNs requires D1R-GluN1} heteromerization. 
To study the function of the D1R-GluN1 heteromers in cocaine-ind uced adaptations, we designed an adenoassociated virus (AAV)-based strategy to disrupt heteromers in a spatially- and temporally-controlled manner (Fig. 2A). The AAV Tet-On-GluN1C1 allows a doxycycline (dox)-inducible bicistronic expression of the RFP reporter protein together with a peptide corresponding to the C1 cassette ( $\left.\mathrm{D}_{864}-\mathrm{T}_{900}\right)$ of GluN1 that binds to D1R (28). This peptide blocks D1R-GluN1 interaction in vitro, while preserving the functions of individual D1R and NMDAR independently of their heteromerization (31). The control virus, Tet-OnGluN1C1 $\Delta$, encodes a $\mathrm{C} 1$ cassette deleted of 9 amino acids that are required for electrostatic interactions between D1R and GluN1 (38). This mutated cassette does not interfere with D1R-GluN1 interaction in vitro (31). After stereotaxic injections in the NAc, the treatment with dox triggered a rapid and sustained expression of RFP (Fig. 2A). In naive mice, analysis of D1R-GluN1 proximity in RFP-positive neurons showed that Tet-On-GluN1C1 significantly reduced D1R-GluN1 PLA puncta when compared to the control AAV (Fig. 2B,C), indicating an efficient disruption of these heteromers in vivo. We also verified that blocking D1R-GluN1 heteromerization altered downstream cocaine-mediated signaling events (20, 31), including GluN2B phosphorylation and extracellular-signal regulated kinase (ERK) pathway activation (Fig. S2), without compromising neuronal survival (Fig. 2D).

Long-lasting changes of glutamate transmission at cortical projections onto D1R-MSN of the NAc have been causally implicated in the development of cocaine-induced locomotor sensitization (13). To study the contribution of D1R-GluN1 heteromerization in drug-induced plasticity at these synapses, we injected mice with Tet-On-GluN1C1 together with a mixture of AAV-PPTA-Cre - driving the expression of the Cre recombinase under the control of the D1R-MSN-specific prepro-tachykinin promoter - and AAV-DIOeGFP to tag D1R-MSN (7, 39, 40) (Fig. S3A). These mice were supplemented with dox before and during daily injections of saline or cocaine for 5 days $(5 \mathrm{~d})$ followed by $10 \mathrm{~d}$ of withdrawal. As previously shown (16), cocaine triggered an increase of AMPA/NMDA $(\mathrm{A} / \mathrm{N})$ ratio - an index of synaptic plasticity - in D1RMSN of mice injected with the control virus in the NAc. By contrast, while preserving basal synaptic transmission in the saline-treated group, the inhibition of D1R-GluN1 heteromerization blunted the cocaineevoked increase in $\mathrm{A} / \mathrm{N}$ ratio (Fig. 2E,F), without modifying the amplitude or the kinetics of NMDAR EPSCs (Fig. 2G,H). These data show that D1R-GluN1 heteromerization controls cocaine-evoked changes in glutamate transmission in D1R-MSNs.

\section{D1R-GluN1 heteromerization controls the development of cocaine-induced locomotor sensitization}

We next evaluated the role of D1R-GluN1 heteromerization in the behavioral sensitizing properties of cocaine by supplementing Tet-On-AAV-injected mice with dox prior to and during saline or cocaine administration. Uncoupling D1R from GluN1 did not affect basal locomotion (Fig. 2I), nor the acute 
hyperlocomotor response triggered by the first cocaine injection, but fully blocked the development of the behavioral sensitization induced by subsequent injections (Fig. 2J). Of note, dox supplementation did not alter body weight, basal locomotion or behavioral sensitization to cocaine in mice that were not injected with Tet-On viruses (Fig. S4A-C).

By temporally controlling expression of the interfering peptides, we assessed the contribution of D1RGluN1 heteromerization to the maintenance phase of locomotor sensitization. Mice injected with Tet-On viruses were treated with saline or cocaine for $5 \mathrm{~d}$ in the absence of dox. As expected, these mice displayed a similar cocaine-induced locomotor sensitization regardless of the virus injected. To switch off D1RGluN1 heteromerization after behavioral sensitization, dox was given after the last saline or cocaine injection and during a $7 \mathrm{~d}$ withdrawal followed by a challenge injection of saline or cocaine (Fig. 2K-top).

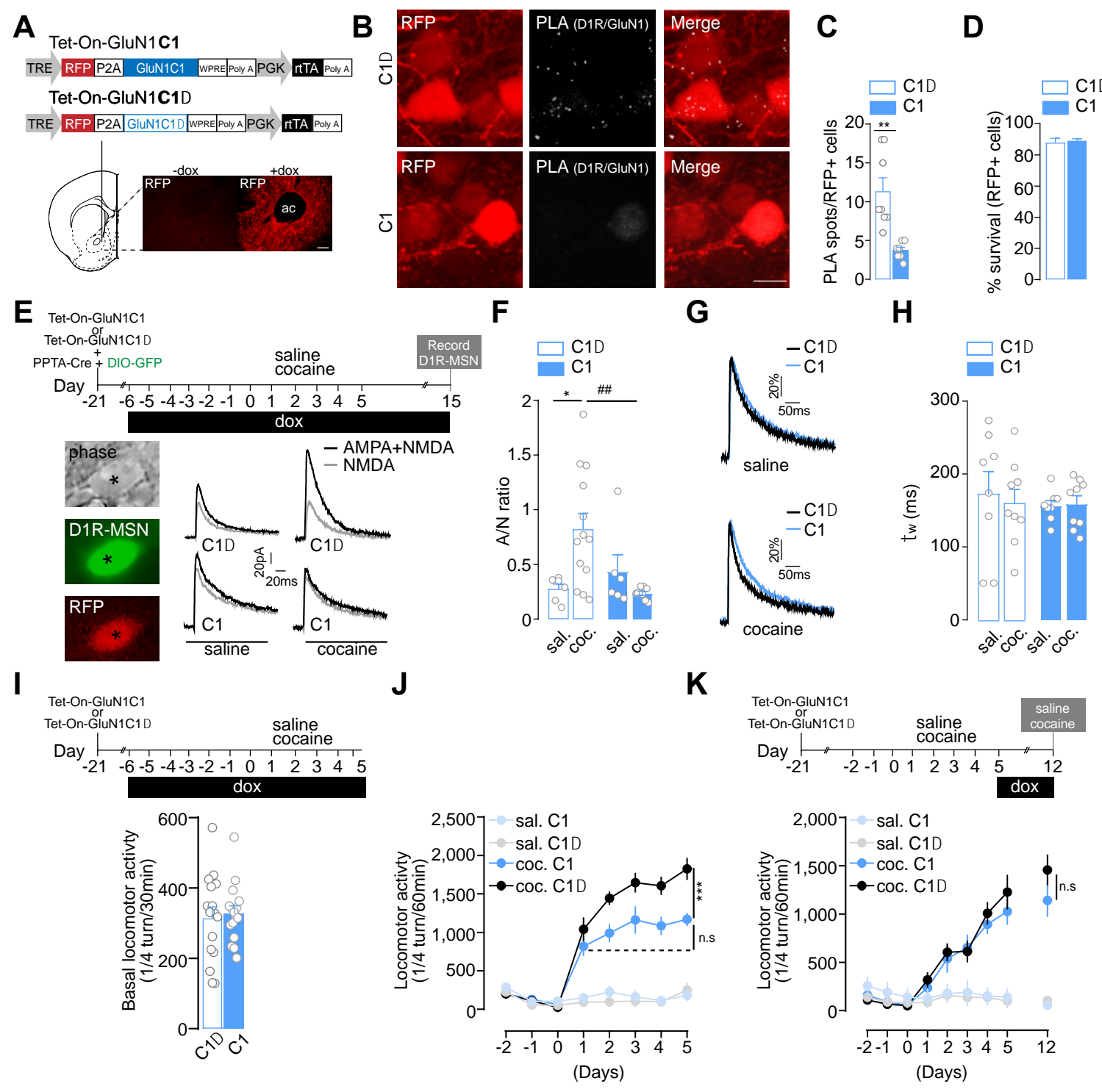


Figure 2. D1R-GluN1 heteromerization controls cocaine-evoked potentiation of glutamate transmission onto D1R-MSN and the development of behavioral sensitization. (A) Top: Viral-based strategy for expression of interfering peptide to disrupt D1R-GluN1 interaction (Tet-On-GluNC1; C1) and control (Tet-On-GluN1C1 $\Delta$; $\mathrm{C} 1 \Delta$ ) in the NAc. Bottom: Example image of doxycycline (+dox)-mediated RFP expression (ac: anterior commissure). (B) Example image of D1R-GluN1 heteromer detection by PLA in C1 $\Delta$ - and C1-transduce neurons. Scale bar: $10 \mu \mathrm{m}$. (C) Quantifications of the PLA signal in C1 $\Delta$ - and C1-transduced neurons. Two-sided Student's t-test, $t=4.078$ df. $=13,{ }^{* *} P=0.0013, \mathrm{n}=7-8$ cells from 4 mice/group. (D) Neuronal survival of $\mathrm{C} 1 \Delta$ - and $\mathrm{C} 1$-transduced neurons. Two-sided Student's t-test, $t=0.354 \mathrm{df}$. $=6, P=0.735, \mathrm{n}=4$ mice/group. (E) Experimental design and example trace of AMPA+NMDA (black) and NMDA (grey) currentsin neurons (asterisk) expressing GFP (i.e. D1R-MSN (see Fig. $\mathrm{S} 3 \mathrm{~A})$ ), and $\mathrm{C} 1$ or $\mathrm{C} 1 \Delta(\mathrm{RFP}+)$. (F) AMPA to NMDA (A/N) ratios. Two-way ANOVA: virus effect, $\mathrm{F}(1,30)=2.511$, $* \mathrm{P}=0.033$; \#\# $\mathrm{P}=0.0061 ; \mathrm{n}=3-4$ mice/group and $\mathrm{n}=6-13$ cells/group. (G) Comparison of representative recordings of pharmacologically-isolated NMDAR EPSCs, normalized to the peak amplitude (in \%). (H) Deactivation kinetics of NMDAR EPSCs. Two-way ANOVA: virus effect, $\mathrm{F}(1,30)=0.205, \mathrm{P}>0.999, \mathrm{n}=3-4$ mice/group and $\mathrm{n}=8-9$ cells/group. (I) Experimental time frame and basal locomotor activity. Two-sided Student's t-test, $t=0.332 \mathrm{df}$. $=30$, $P=0.742$, n=16 mice per group. (J) Inhibition of D1 R-GluN1 heteromerization during the development of locomotor sensitization. Three-way ANOVA: virus effect, $\mathrm{F}(1,256)=13.72$, *** $\mathrm{P}=0.0003 ; \mathrm{n} . \mathrm{s} \mathrm{P}>0.9999, \mathrm{n}=7-11$ mice/group. (K) Experimental time frame and measurement of locomotor activity in each group. Three-way ANOVA: virus effect, $\mathrm{F}(1,243)=0.6160, \mathrm{P}>0.9999, \mathrm{n}=7-8$ mice/group. n.s not significant. Error bars denote s.e.m.

We found that mice displayed the same level of sensitization in response to the cocaine challenge regardless of the AAV used, demonstrating that D1R-GluN1 heteromerization is not required for the maintenance of locomotor sensitization (Fig. 2K-bottom). Since cocaine also enhanced D1R-GluN1 heteromerization in the dorsal striatum (Fig. 1C), we also targeted heteromers in this striatal sub-region and obtained the same results (Fig. S4D,E). Overall, these data show that D1R-GluN1 heteromerization in the striatum controls the development, but not the maintenance, of cocaine's sensitizing effects.

\section{D2R-GluN2B heteromerization selectively controls the maintenance of cocaine sensitizing effects}

In light of the significant and persistent impact of cocaine on D2R-GluN2B heteromerization in the NAc (see Fig. 1D,E), we generated AAV Tet-On-D2R-IL3 to achieve a dox-inducible expression of a peptide corresponding to a small fragment $\left(\mathrm{T}_{225}-\mathrm{A}_{234}\right)$ located within the $3^{\text {rd }}$ intracellular loop (IL3) of D2R. This IL3 domain is known to play a key role for D2R-GluN2B interaction (32). Since critical amino acids responsible for D2R-GluN2B interaction have not been yet identified within this D2R-IL3 fragment, we used a control virus (Tet-On-D2R-IL3-scr) driving the expression of a scrambled peptide (Fig. 3A). The 
Tet-On-D2R-IL3 efficiently reduced D2R-GluN2B PLA puncta and preserved neuronal survival (Fig. 3BD). Importantly, this peptide altered the interaction of D2R with GluN2B while sparing the functions of individual component receptors, as shown by its lack of effect on D2R-mediated inhibition of cAMP production (Fig. 3E) and NMDA currents (see below).
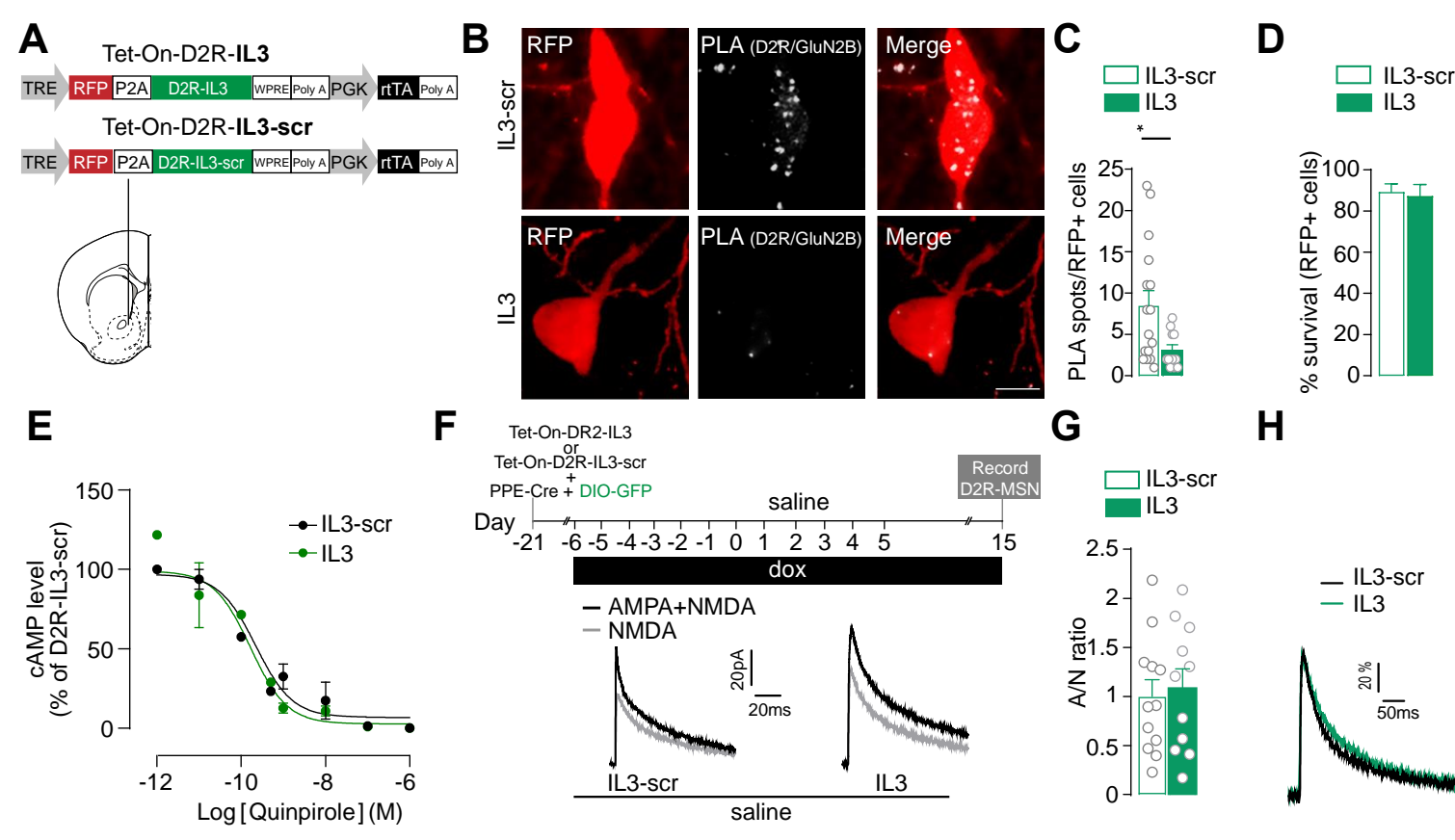

F Tet-On-DR2-IL3

G

H
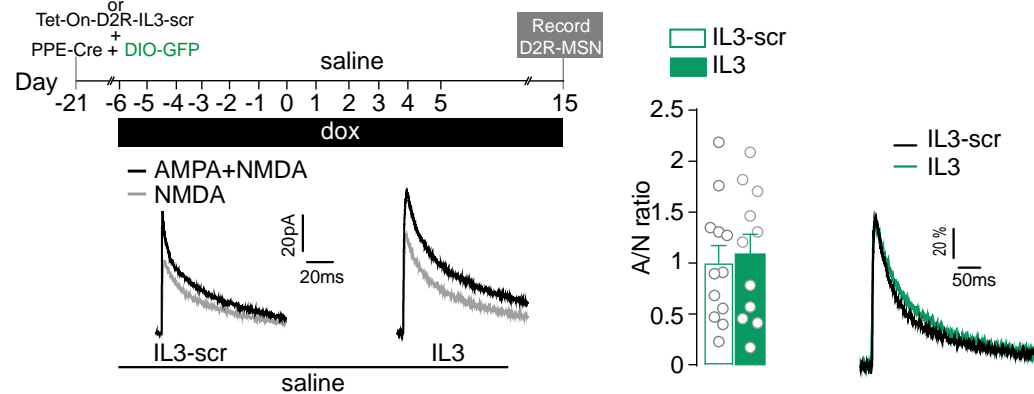

I
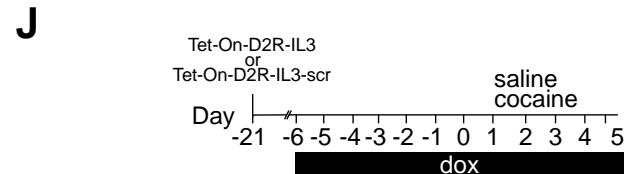

K

Tet-On-DR2-IL3
Tet-On-D2R-IL3-scr Tet-On-D2R-IL3-scr
PPE-cre + DIO-GFP
Day $\left.\begin{array}{cc}500 \\ \frac{0}{3} & 300 \\ 3 & 200\end{array}\right]$
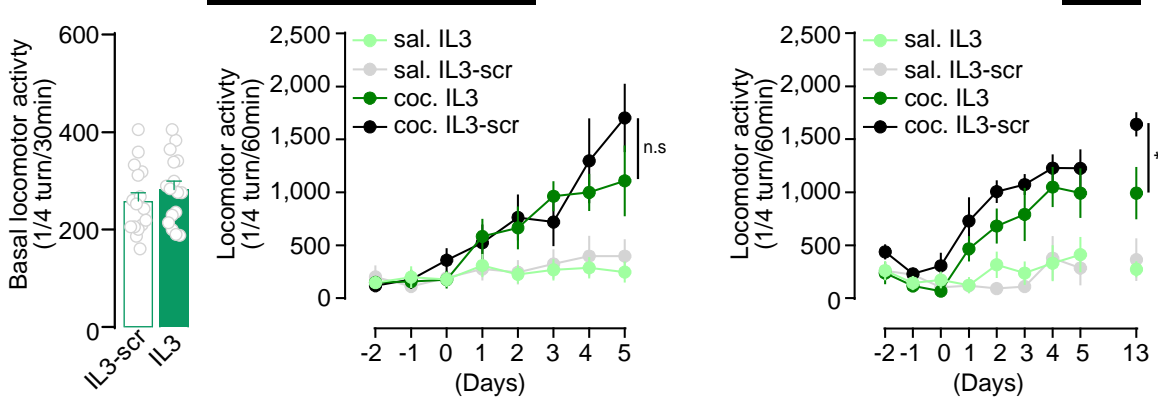

L

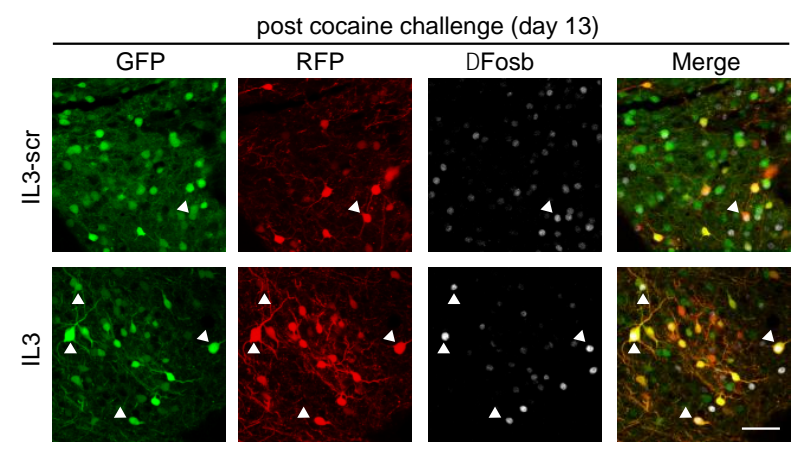

M

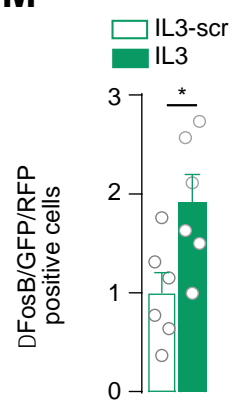

Figure 3. D2R-GluN2B heteromerization selectively controls the maintenance of cocaine sensitizing effects. (A) Viral strategy for expression of interfering peptide to prevent D2R-GluN2B heteromerization (Tet-On-D2R-IL3; IL3) 
and control (Tet-On-D2R-IL3-scr; IL3-scr). (B) Representative image D2R-GluN2B heteromer detection by PLA in IL3-scr- and IL3-transduced neurons. Scale bar: $10 \mu \mathrm{m}$. (C) Quantifications of the PLA signal in IL3-scr- and IL-3 infected neurons. Two-sided Student's t-test, $\mathrm{t}=2.393 \mathrm{df}$. $=25, * * \mathrm{P}=0.0246, \mathrm{n}=11-16$ cells from 4 mice/group. (D) Neuronal survival of IL3-scr- and IL3-transduced neurons. Two-sided Student's t-test, $\mathrm{t}=0.2767 \mathrm{df}$. $=6, \mathrm{P}=0.7913$, $\mathrm{n}=4$ mice/group. (E) Tet-On-D2R-IL3 spares quinpirole-induced inhibition of forskolin-induced accumulation of cAMP. $\log \mathrm{IC}_{50}$ is -9.78 for D2R-IL3 and -9.65 for D2R-IL3-Scr. $\mathrm{n}=3$ independent experiments/condition. (F) Experimental time frame and representative traces of AMPA+NMDA (black) and NMDA (grey) currents in neurons expressing GFP (i.e. D2R-MSN (see Figure S3B)) and IL3 or IL3-scr (RFP+). (G) AMPA to NMDA (A/N) ratios; Two-sided Student's t-test, $\mathrm{t}=0.3397 \mathrm{df} .=21, \mathrm{P}=0.7375, \mathrm{n}=4-6$ mice/group and $\mathrm{n}=11-12$ cells/group. (H) Comparison of representative recordings of pharmacologically-isolated NMDAR EPSCs, normalized to the peak amplitude (in \%). (I) Deactivation kinetics of NMDA EPSCs, Two-sided Student's t-test, t=0.5129 df. $=21$, $\mathrm{P}=0.6134, \mathrm{n}=11-12$ cells/group. (J) Top: Experimental time frame. Bottom left: basal locomotor activity; Two-sided Student's t-test, $\mathrm{t}=0.994 \mathrm{df} .=30, \mathrm{P}=0.3282, \mathrm{n}=16$ mice/group. Bottom right: Inhibition of $\mathrm{D} 2 \mathrm{R}-\mathrm{GluN} 2 \mathrm{~B}$ heteromerization does not impair the development of locomotor sensitization Three-way ANOVA: virus effect, $\mathrm{F}$ (1, $192)=1.984$, n.s P >0.9999; n=6-8 mice/group. $(\mathbf{K})$ Experimental time frame and impact of D2R-GluN2B heteromer inhibition on the maintenance of cocaine-evoked locomotor sensitization. Three-way ANOVA: virus effect, F (1, $198)=7.278, * \mathrm{P}=0.0330 ; \mathrm{n}=6$ mice/group. $(\mathbf{L})$ Representative images of $\Delta$ FosB expression in D2R-MSN (GFP+) infected with IL3-scr or IL3 (RFP+) after the cocaine challenge injection (see panel K). Arrowheads show GFP+/RFP+/ $\triangle$ FosB+D2R-MSN. Scale bar: $50 \mu \mathrm{m}$. (M) Quantifications of GFP+/RFP+/ $\Delta$ FosB+D2R-MSN. Twosided Student's t-test, $\mathrm{t}=2.694 \mathrm{df} .=10,{ }^{*} \mathrm{P}=0.0225, \mathrm{n}=6 \mathrm{mice} /$ group. $\mathrm{n} . \mathrm{s}$ : not significant. Error bars denote s.e.m.

Since repeated cocaine exposure does not modify A/N ratio in D2R-MSN (16), the consequences of uncoupling D2R from GluN2B was studied in saline-treated animals with virally-tagged D2R-MSN owing to the co-injection of AAV-PPE-Cre - driving the expression of the Cre recombinase under the control of the D2R-MSN-specific prepro-enkephalin promoter (7) and AAV-DIO-eGFP(Fig. S3B). We found that the inhibition of D2R-GluN2B heteromerization did not alter, by itself, A/N ratio in D2R-MSN when compared to D2R-MSN transd uced with the control virus (Fig. 3F,G). Of note, Tet-On-D2R-IL3 also left unchanged the amplitude and kinetics of NMDA currents (Fig. 3H,I), ind icating a lack of non-specific effect on individual NMDAR functions, thereby validating our interfering viral strategy.

At the behavioral level, interfering with D2R-GluN2B heteromerization preserved both basal locomotion (Fig. 3J-left) and the development of cocaine-induced locomotor sensitization (Fig. 3J-right).

Strikingly, we found that the inhibition of D2R-GluN2B heteromerization during cocaine withdrawal reduced the maintenance of the behavioral sensitization compared to cocaine-treated mice injected with the 
control virus (Fig. $3 \mathrm{~K}$ ). These mice were sacrificed to analyze $\Delta$ FosB expression levels, used here as a proxy for neuronal activity. We observed a significant increase of D2R-MSN expressing $\Delta$ FosB after the cocaine challenge upon inhibition of D2R-GluN2B interaction (Fig. 3L,M). Since D2R-Glu2NB heteromerization has been shown to mediate the D2R agonist-induced inhibition of NMDAR ex vivo (32), our data suggest that the impaired maintenance of the sensitizing effects of cocaine observed upon D2RGluN2B uncoupling may result from increased D2R-MSN activity.

Altogether, these data demonstrate that D1R-GluN1 and D2R-GluN2B heteromerization controls the development and maintenance of cocaine sensitizing effects, respectively.

\section{Differential roles of D1R-GluN1 and D2R-GluN2B heteromers in the rewarding effects of cocaine.}

We next investigated the role of these heteromers in the rewarding effects of cocaine using a conditioned place preference (CPP) paradigm. Mice injected in the NAc with a control virus and supplemented with dox developed a significant cocaine-induced CPP, which was blunted when D1R and GluN1 were uncoupled (Fig. 4A). Although single or repeated cocaine administration has been shown to trigger dendritic spine formation in D1R-MSN $(39,41)$, there is no study showing such morphological changes in the NAc in the context of CPP. Mice were thus sacrificed the day after the behavioral test to perform a 3D morphological analysis of GFP-tagged D1R-MSN. Control mice, which developed CPP to cocaine, displayed a significant increase of dendritic spine density in D1R-MSN, which was inhibited when D1R and GluN1 were uncoupled (Fig. 4B). To study the implication of D1R-GluN1 heteromerization on relapse to CPP, AAV-injected mice were initially trained for CPP in the absence of dox. Once mice developed CPP, dox was added to alter D1R-GluN1 interaction during an extinction period followed by a cocaine-induced relapse. We observed that the inhibition of D1R-GluN1 interaction did not alter the kinetics of extinction, nor the relapse to CPP (Fig. 4C), thus supporting a critical role for D1R-GluN1 heteromers in the development of the rewarding effects of cocaine, as observed for locomotor sensitization, but not in the propensity to relapse.

The uncoupling of D2R from GluN2B also blocked the development of cocaine CPP (Fig. 4D) but this was not correlated to morphological changes in D2R-MSNs since the CPP paradigm did not trigger any modification of dendritic spine density in D2R-MSN regardless of the AAV used (Fig. 4E). Inhibiting D2RGluN2B heteromerization once the mice have developed CPP did not impact the extinction of CPP but significantly reduced cocaine-induced relapse (Fig. 4F). This indicates that D2R-GluN2B heteromerization is required for both the development and relapse of cocaine-induced CPP, independently of morphological changes in GFP-tagged D2R-MSNs. 


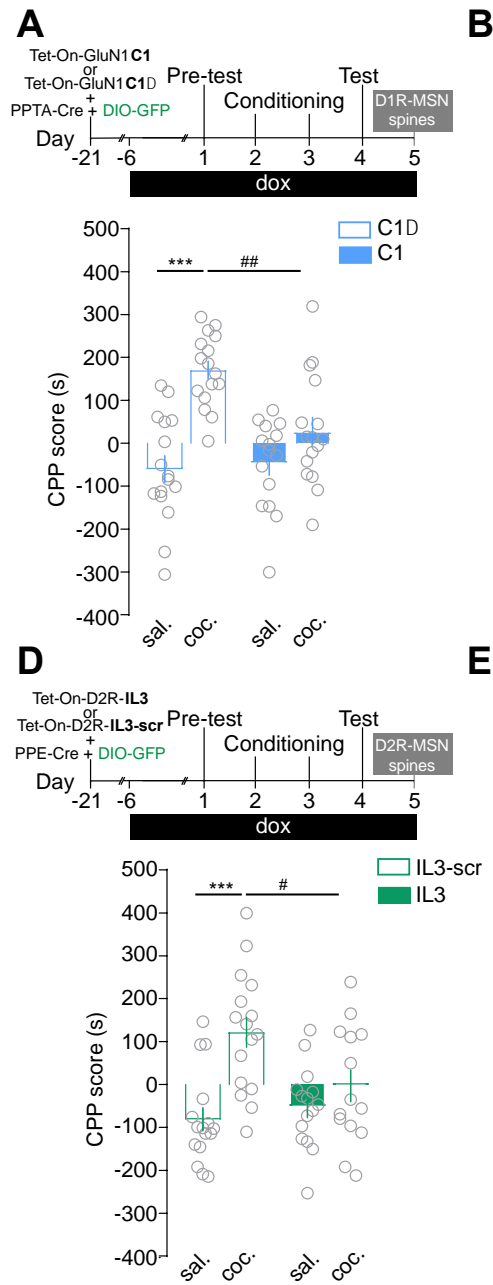

B
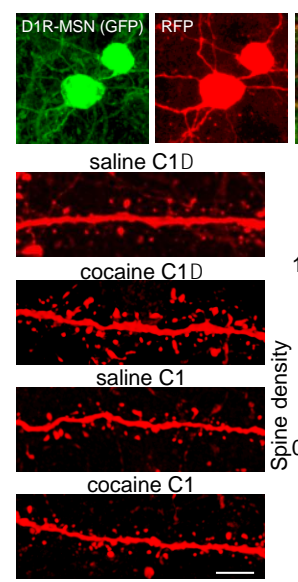

E
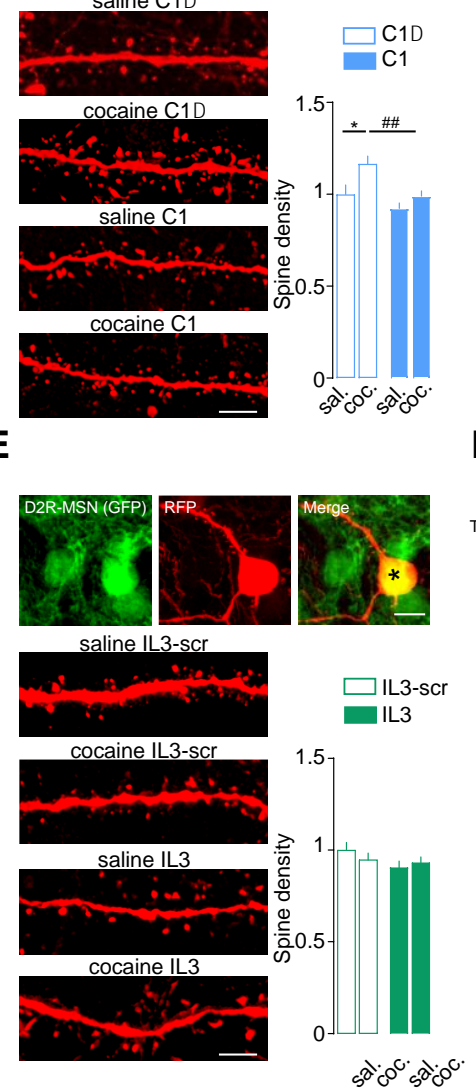

C
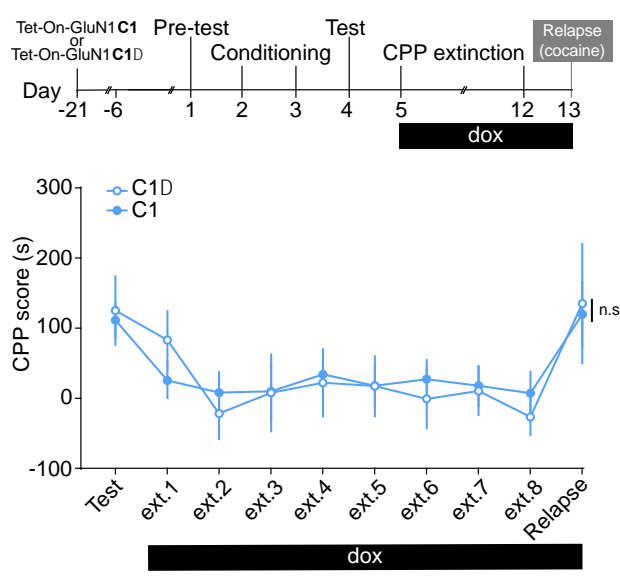

$\mathbf{F}$
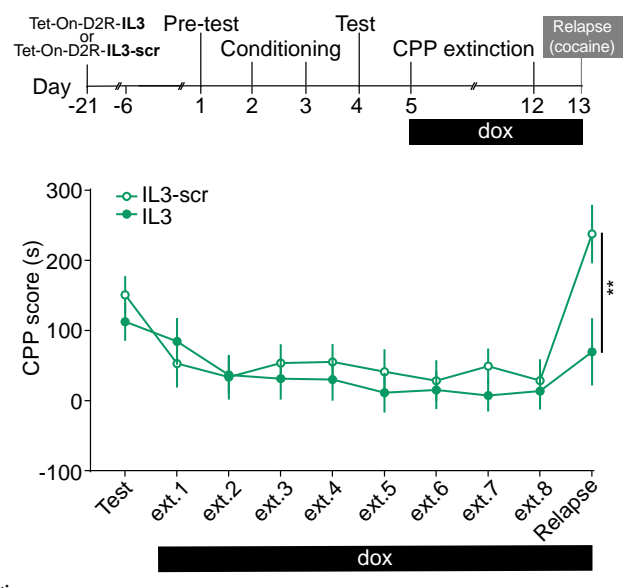

Figure 4. Differential roles of D1R-GluN1 and D2R-GluN2B heteromerization in controlling the rewarding effects of cocaine. (A) Experimental time frame and conditioned place preference (CPP) score upon inhibition of D1R-GluN1 heteromerization. Two-way ANOVA: virus effect, $\mathrm{F}(1,59)=5.281$, *** $\mathrm{P}<0.0001$; \#\# $\mathrm{P}=0.0040, \mathrm{n}=15$ 16 mice/group. (B) Top: low magnification images of D1R-MSN (GFP+; see Fig. S3A) infected (RFP) shown by the asterisks Scale bar $10 \mu \mathrm{m}$. Bottom: high magnification of dendritic segments. Scale bar: $5 \mu \mathrm{m}$. Spine density analysis. Two-way ANOVA: virus effect, $\mathrm{F}(1,162)=11.14,{ }^{*} \mathrm{P}=0.0446$; \#\# $\mathrm{P}=0.0043, \mathrm{n}=27$-69 dendrites from 6 mice/group. (C) Experimental time frame to study the impact of D1R-GluN1 uncoupling on the extinction and cocaine inducedrelapse to CPP. Two-way ANOVA: virus effect, $\mathrm{F}(1,24)=0.004, \mathrm{P}>0.999$, cocaine $\mathrm{C} 1$ vs cocaine $\mathrm{C} 1 \Delta$, CPP score on relapse day, $\mathrm{n}=10-16$ mice/group. (D) Same as for (A) upon inhibition of D2R-GluN2B heteromerization. Twoway ANOVA: virus effect, $\mathrm{F}(1,57)=2.424,{ }^{* * *} \mathrm{P}<0.0001 ;{ }^{\#} \mathrm{P}=0.0396, \mathrm{n}=14-16$ mice per group. $(\mathbf{E})$ Top: low magnification images of D2R-MSN (GFP; see Fig S3B) infected (RFP) shown by the asterisk. Scale bar $10 \mu \mathrm{m}$. Bottom: high magnification of dendritic segments. Scale bar: $5 \mu \mathrm{m}$. Spine density analysis. Two-way ANOVA: virus effect, $\mathrm{F}(1,166)=0.1268$, n.s P >0.999 saline IL3-scr vs cocaine IL3-scr; n=30-53 dendrites from 6 mice/group. (F) 
CPP score upon inhibition of D2R-GluN2B during CCP during extinction and cocaine-induced relapse. Two-way ANOVA: virus effect, $\mathrm{F}(1,31)=0.899,{ }^{* *} \mathrm{P}=0.0018$, cocaine IL3 vs cocaine IL3 - scr CPP score on relapse day, $\mathrm{n}=16-17$ mice/group. n.s: not significant. Error bars denote s.e.m.

\section{Inhibiting D1R-GluN1 or D2R-GluN2B heteromerization does not alter conditioned place preference} for food.

Manipulating D1R-GluN1 or D2R-GluN2B heteromerization in vivo allowed us to reveal their selective implication in controlling distinct phases of long-term cocaine-evoked adaptations. We next examined whether receptor heteromerization also controls non-drug reward processing. We found that disrupting either heteromer subtype, using comparable conditions as for our studies with cocaine, failed to alter the rewarding properties of food (Fig. 5), supporting a role of these heteromers in controlling the rewarding effects of cocaine but not a non-drug reward.

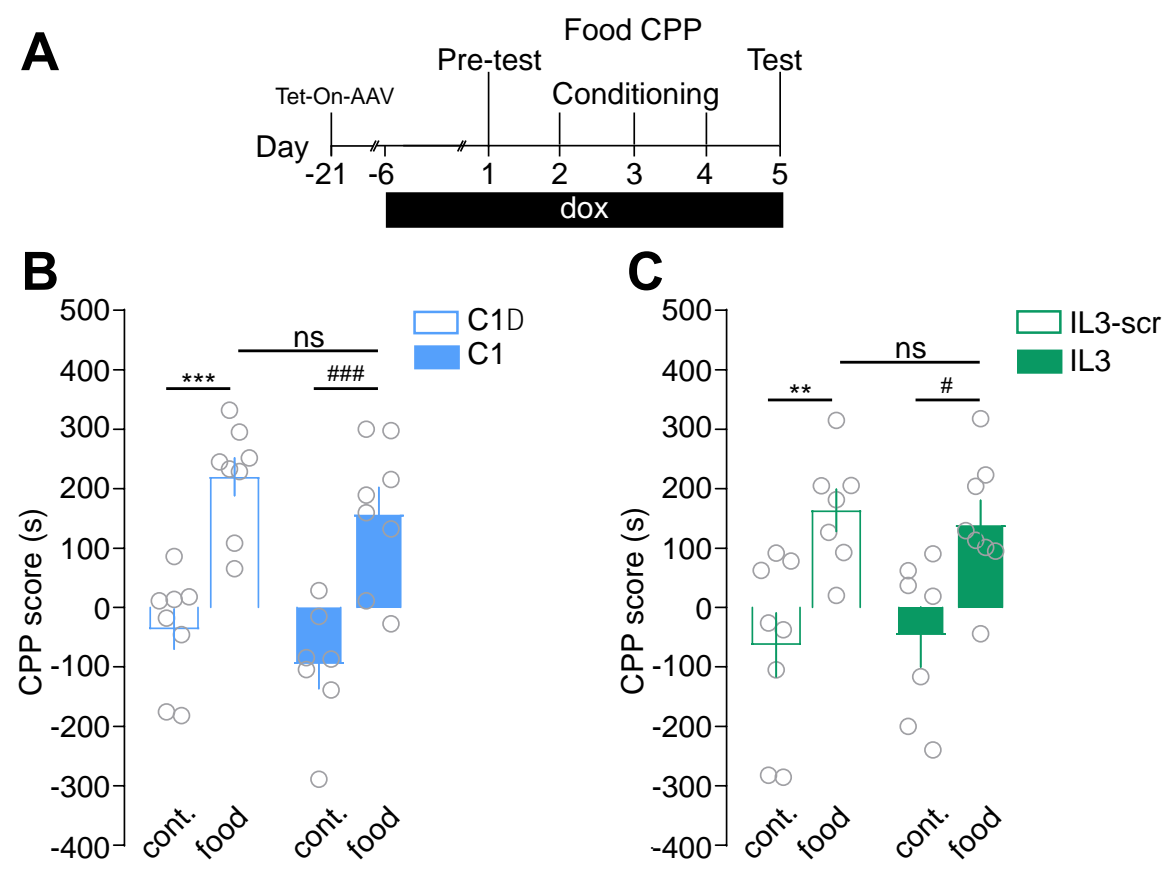

Figure 5. Inhibiting D1R-GluN1 or D2R-GluN2B heteromerization does not alter conditioned place preference

for food. (A) Experimental time frame to study the consequences of inhibition of heteromerization on the development of food-induced CPP. (B) Impact of inhibiting D1 R-GluN1 heteromerization on the CPP score. Two-

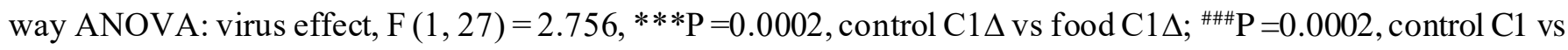
food $\mathrm{C} 1$; n.s $\mathrm{P}>0.999$, food $\mathrm{C} 1$ vs food $\mathrm{C} 1 \Delta, \mathrm{n}=7-8$ mice/group. (C) Effect of inhibiting D2R-GluN2B heteromerization on the CPP score. Two-way ANOVA: virus effect $\mathrm{F}(1,26)=0.007,{ }^{* *} \mathrm{P}<0.0098$, control IL3-scr vs food IL3-scr; "P =0.0366, control IL3 vs food IL3; n.s, P>0.999 food IL3 vs food IL3 -scr, n=7-8 mice/group. n.s: not significant. Error bars denote s.e.m. 


\section{D2R-GluN2B heteromerization is increased in post-mortem brain samples from addict subjects despite decreased D2R expression.}

As evidenced above, D2R-GluN2B heteromerization plays a cardinal role in the maintenance of cocaine's effects without affecting natural reward processing in mice, which positions this heteromer subtype as a potential therapeutic target for drug addiction. We therefore investigated whether D2R-GluN2B heteromerization could be detected in human brain tissues and modulated in subjects with a history of psychostimulant dependence.

PLA has recently been shown as a suitable approach to detect single proteins or receptor heteromers, including D2R-A2AR, in human brain samples $(42,43)$. We therefore performed single detection of $\mathrm{D} 2 \mathrm{R}$, GluN2B and D2R-GluN2B heteromers in post-mortem human samples from control subjects and matched individuals with a history of dependence. Although often poly-addicts, these individuals were selected for their main dependence to psychostimulants and the presence of traces of psychostimulants in their blood at the time of death (Table S1). From whole-slide images of caudate putamen samples, automated detection of PLA signal was performed from 25 high-magnification images per subjects randomly selected within the ventral part of the samples, which corresponds to the mouse NAc (Fig. S5).

D2R single detection produced a dense punctate pattern in control subjects (Fig. 6A), as already reported (42). As expected, this signal was absent when PLA was performed in the absence of the primary antibody. Interestingly, we detected a significant decrease of relative D2R protein expression in sample from addicts compared to control subjects (Fig. 6B), consistent with the well-established decrease of striatal D2R availability reported in psychostimulant abusers by PET imaging (44-48). By contrast, GluN2B single detection produced a dense GluN2B signal that was not different between samples from ad dicts and controls (Fig. 6C,D). The double recognition of D2R-GluN2B proximity yielded a punctate signal in control subjects, which was undetectable when one of the two primary antibodies was omitted (Fig. 6E).

In samples from addicts, there was a trend towards an increase of D2R-GluN2B heteromers in samples from addicts (Fig. 6F), despite the drastic decrease of D2R levels. We therefore analyzed whether a correlation between D2R levels and D2R-GluN2B heteromerization could exist within each addict sample. We found a significant inverse correlation between these two parameters (Fig. 6G), suggesting that despite the lower levels of D2R expression in addicts, at least a remaining pool of D2R was preferentially involved in D2RGluN2B heteromerization. By contrast, there was no correlation between GluN2B expression and D2RGluN2B heteromerization (Fig. 6F). Considering the decreased D2R levels in samples from addicts, we normalized the D2R-GluN2B PLA signal to D2R levels for each individual and found a significant increase in relative D2R heteromerization with GluN2B in samples from addicts when compared to controls (Fig. 
6I). To the best of our knowledge these results provide the first evidence of a decreased D2R protein expression in the striatum of psychostimulant addicts, which is associated with an increase of D2R-GluN2B heteromerization. Together with our interventional approach in mice, our data support D2R-GluN2B heteromers as therapeutic targets of potential interest in addiction.

A
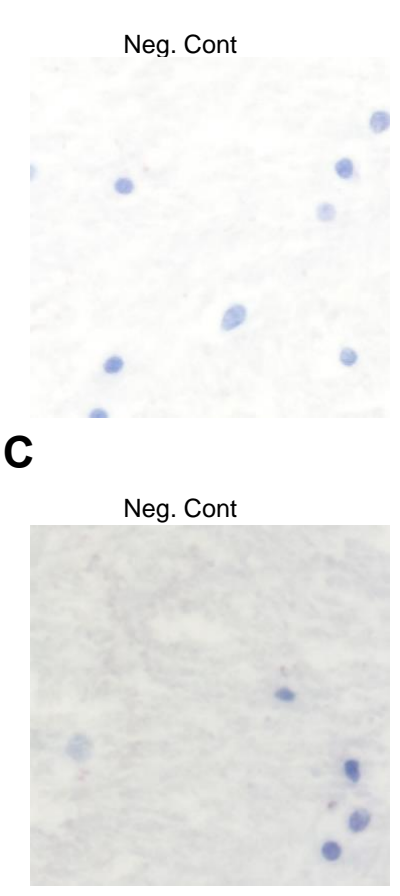

E

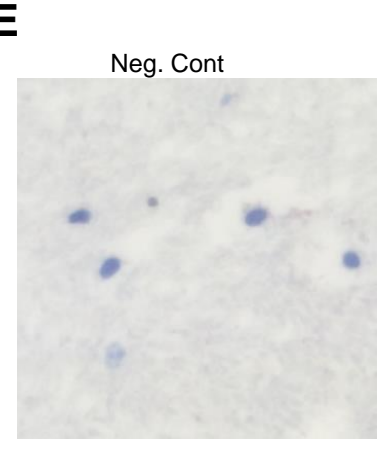

G

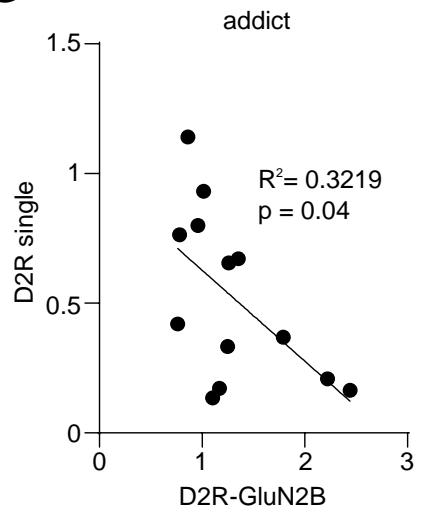

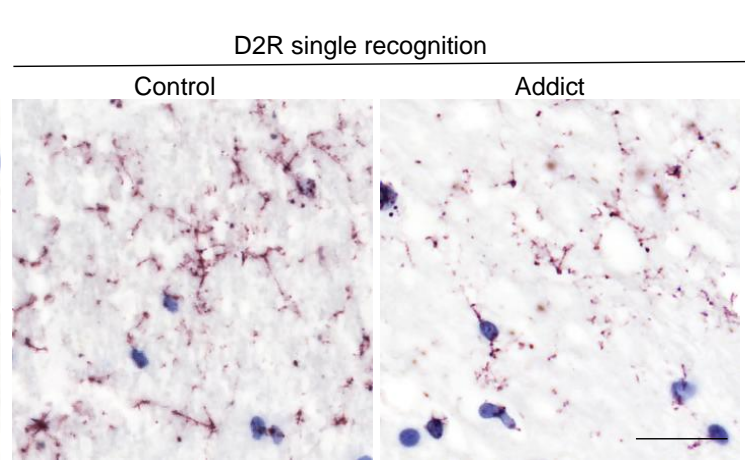

GluN2B single recognition

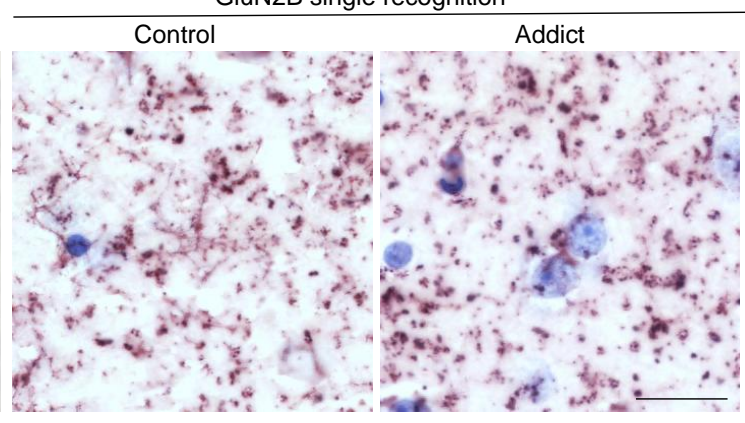

D2R-GluN2B

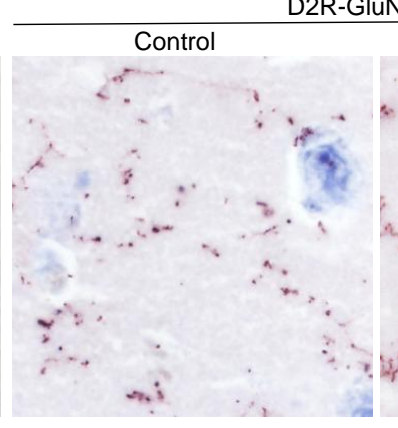

H

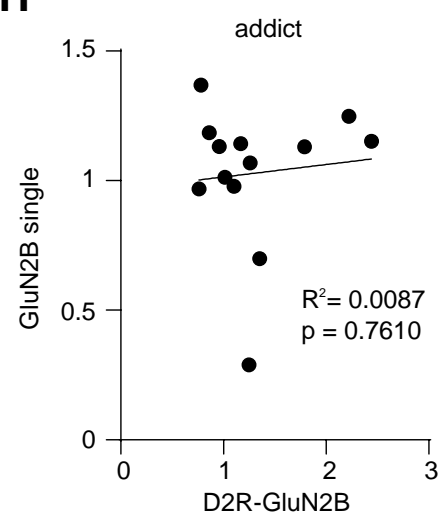

I
B

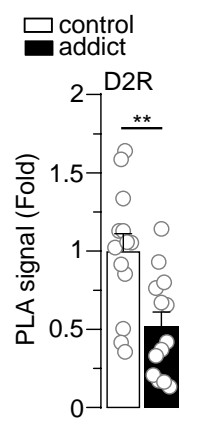

D

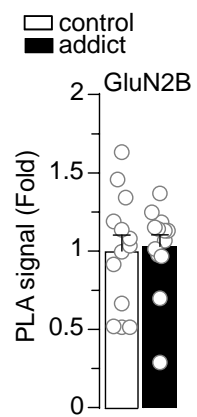

$F$

$\square$ control

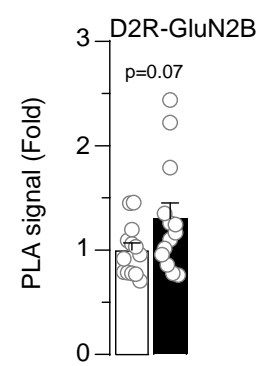

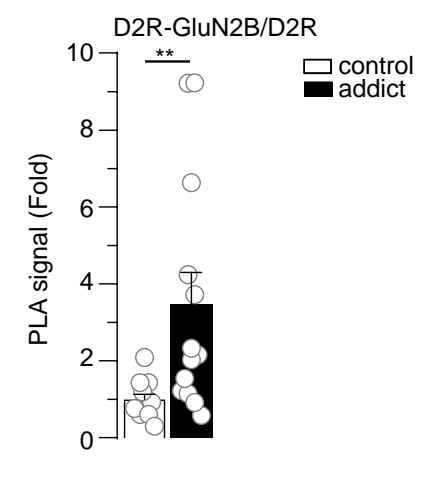


Figure 6 D2R-GluN2B heteromerization is increased in post-mortem brain samples from addict subjects despite decreased D2R expression. (A) Representative images of D2R single recognition by PLA and negative control, in which the primary antibody is omitted (left panel Neg. Cont; see Fig. S5). (B) Quantifications of D2R single PLA signal represented as fold decreased compared to control subjects. Two-sided Student's $\mathrm{t}$-test, $\mathrm{t}=3.331$ df. $=24,{ }^{* *} \mathrm{P}=0.0028, \mathrm{n}=13$ subjects/group. (C) Example images of GluN2B single detection and Neg. Cont. (D) Quantifications of Glu2NB PLA signal. Two-sided Student's t-test, $\mathrm{t}=0.224 \mathrm{df}$. $=24, \mathrm{P}=0.8243, \mathrm{n}=13$ subjects/group. (E) Illustrative images of D2R-GluN2B heteromer detection by PLA and Neg. Cont (GluN2B antibody omitted). (F) Quantifications of D2R-GluN2B PLA signal. Two-sided Student's t-test, $\mathrm{t}=1.868 \mathrm{df}$. $=24$, $\mathrm{P}=0.074, \mathrm{n}=13$ subjects per group. (G) Pearson correlation between D2R expression levels and D2R-GluN2B heteromerization for each sample from all addict subjects, $\mathrm{R}^{2}=0.3219, \mathrm{P}=0.0432$. (H) Pearson correlation between GluN2B expression levels and D2R-GluN2B heteromerization for each sample from all addict subjects $\mathrm{R}^{2}=0.0087$, $\mathrm{P}=$ 0.761. (I) Quantifications of D2R-GluN2B PLA signal normalized to D2R expression levels for each subject. Two-sided Student's t-test, $\mathrm{t}=2.882 \mathrm{df} .=24,{ }^{*} \mathrm{P}=0.0082, \mathrm{n}=13$ subjects/group. (A, C, E) Scale bar: $25 \mu \mathrm{m}$. Error bars denote s.e.m.

\section{Discussion}

Optogenetic studies undeniably showed that distinct phases of drug-induced behavioral adaptations rely on DA-evoked synaptic adaptations at specific glutamate inputs onto MSN subpopulations (13-15, 49, 50). Nonetheless, the underlying molecular mechanisms remain poorly understood (2). This is an important issue because the identification of events responsible for such a detrimental interplay between dopamine and glutamate signaling may help in the development of innovative strategies with therapeutic potential. Herein, we provide multiple lines of evidence, from mice to humans, that the heteromerization of glutamate NMDAR with D1R or D2R is enhanced by psychostimulants and preferentially controls the development and maintenance phases of cocaine-evoked long-term adaptations, respectively.

The focus on dopamine and NMDA receptor heteromers as potential integrators of dopamine and glutamate inputs that may control drug-mediated adaptations stems from in vitro and ex vivo studies showing that such a direct physical interaction allows a reciprocal fine-tuning of the component receptors' functions $(23,27)$. In particular, patch-clamp recording from striatal slices showed that D1R-GluN1 and D2R-GluN2B interactions respectively facilitate and inhibit NMDAR-mediated signaling upon DA increase (31, 32). An appealing hypothesis would therefore be that, by linking dopamine to glutamate signaling in opposite ways, these heteromers could constitute molecular substrates for drugs of abuse to exert their differential effects on the activity of MSN subtypes, which has been proposed to underlie the switch from recreational drug consumption to addiction $(3,51)$. 
In agreement with this model, our PLA analysis showed that locomotor sensitization induced by repeated cocaine injections was associated with an increase of both heteromers in the NAc. We also found that this increased heteromerization requires dopamine receptor stimulation. While the PLA method cannot establish the direct physical contact of the two proteins or the stoichiometry of the complex, it does indicate that the proteins are in close molecular proximity (34). Nonetheless, previous studies have provided evidence for direct interactions between the D1R-GluN1 and D2R-GluN2B $(28,32)$ and we find that our viral minigenes selectively decrease the PLA signals; we therefore interpret these PLA data as support for receptor heteromerization. While in-depth characterization of the molecular events responsible for this increased receptor interaction upon cocaine exposure is beyond the scope of this study, a possible explanation may lie in the observation that repeated cocaine exposure decreases PSD-95 expression in the NAc (36). In fact, PSD-95 is a known endogenous inhibitor of D1R-GluN1 interaction (37) that also binds to D2R (52) and the GluN2B c-terminal end (53). Even though the PLA approach is able to provide a snapshot of the impact of cocaine on receptor heteromerization in situ in their native environment $(31,34,35)$, future work is needed to investigate whether heteromerization of DA and NMDA receptors is an input-specific process and whether it relies on the modulation of receptor surface expression and/or dynamics.

The development of the sensitizing effects of cocaine has been previously causally linked to the potentiation of glutamate transmission at cortical projections onto D1R-MSN of the NAc (13). Since we observed that preventing D1R-GluN1 heteromerization reversed both alterations in $\mathrm{A} / \mathrm{N}$ ratio and the development of behavioral sensitization, while sparing the function of individual component receptors (see (31)), our results suggest that D1R-GluN1 heteromers are key molecular platforms for the development of cocaine-induced long-term adaptations. In contrast, disrupting D1R-GluN1 interaction during a withdrawal from cocaine did not impact maintenance of the sensitized state, supporting a preferential role of this heteromer subtype in the initial phases of cocaine-mediated adaptations. In agreement with this hypothesis, we observed that preventing D1R-GluN1 heteromerization during CPP conditioning also blocked the development of the rewarding effects of cocaine, but failed to alter the extinction and relapse phases. This critical time window of D1R-GluN1 heteromer function restricted to the early developmental phase of cocaine-evoked adaptations agrees with our observation that cocaine-induced D1R-GluN1 heteromerization is a transient mechanism that does not outlast a $7 \mathrm{~d}$ withdrawal period. Instead, the temporally-controlled disruption of D2R-GluN2B heteromers revealed their preferential role in the maintenance of the sensitizing and rewarding effects of cocaine. In agreement with these findings, we found that D2R-GluN2B heteromerization persisted through withdrawal from cocaine. Moreover, this persistent heteromerization was specifically observed in the NAc core, which has been identified as a common output structure of neuronal circuits involved in both cue- and drug-induced relapse (54). Since the optogenetic activation of 
D2R-MSNs in the NAc has been shown to preserve cocaine-induced locomotor sensitization but to blunt its expression after withdrawal (55), our results support a model by which inhibiting endogenous D2RGluN2B interaction during withdrawal hinders the persistence of cocaine-evoked responses by potentiating D2R-MSN activity. In support of this hypothesis, we found that the alteration of the maintenance phase of locomotor sensitization observed upon D2R-GluN2B heteromer disruption was associated with an increase of D2R-MSN activity, as revealed by an increased expression of $\Delta$ FosB in D2R-MSN. These observations therefore suggest that D2R-GluN2B heteromerization is a key molecular mechanism triggered by cocaine that dampens D2R-MSN activity and contributes to the persistence of cocaine-evoked adaptations.

Direct manipulations of MSN activity have clearly revealed that D1R-MSN and D2R-MSN activation, respectively, facilitates and blunts the development and maintenance phases of psychostimulant-induced behavioral adaptations (2). Strikingly, our findings that D1R-GluN1 and D2R-GluN2B heteromerization are involved in the induction and maintenance of cocaine-induced locomotor sensitization, respectively, highlight that these receptor complexes mediate discrete properties of MSN subpopulations and play complementary roles to mediate the full panel of cocaine-induced adaptations. Importantly, in further support of specific functions of dopamine and glutamate receptor heteromers, we established that their roles in shaping reward processing depends on the nature of the reward, since the disruption of either receptor heteromer blocked the development of cocaine-induced CPP but spared food-mediated CPP. Although the mechanisms underlying such selectivity to drug reward remain to be established, our findings suggest that targeting dopamine-glutamate receptor heteromers has the potential to preferentially alleviate pathological adaptations induced by drugs of abuse. In particular, the role for D2R-Glu2NB heteromerization in maintaining cocaine-induced adaptations combined with its lack of implication in reward processing to a natural reinforcer suggest that D2R-GluN2B heteromers are targets of choice from a translational standpoint. This led us to investigate whether this heteromer subtype could be detected in post-mortem human samples and modulated in subjects with a history of psychostimulant addiction.

Our PLA analysis revealed a strong reduction of D2R protein levels in the NAc of drug abusers. With the "single PLA" approach, the polyclonal secondary antibodies can bind to either a single primary antibody therefore detecting a single antigen on the $\mathrm{D} 2 \mathrm{R}$ - or to two different primary antibodies bound to proximal antigens - potentially revealing D2R homodimers. However, the latter is likely to be much less efficient and we assume that the single PLA signal in our study mainly reflects the density of single D2R (42). This first observation of a decreased D2R protein expression in post-mortem brain samples from addicts is consistent with the downregulation of $\mathrm{D} 2 \mathrm{R}$ mRNA levels that has been described after long-term cocaine exposure in rats (56). Importantly, this finding could also partly account for the decrease in D2R binding readily observed with PET imaging of the striatum of drug abusers (44-48). Despite such downregulation 
of $\mathrm{D} 2 \mathrm{R}$ protein, the proportion of $\mathrm{D} 2 \mathrm{R}$ forming heteromers with GluN2B was three-fold higher in psychostimulant abusers compared to healthy subjects. Strikingly, addict individuals bearing the lowest $\mathrm{D} 2 \mathrm{R}$ expression displayed the highest density of $\mathrm{D} 2 \mathrm{R}-\mathrm{GluN} 2 \mathrm{~B}$. This raises questions regarding the underlying molecular mechanism of D2R-GluN2B formation in response to psychostimulant exposure in human. Based on our findings in mice that cocaine-induced D2R-GluN2B heteromerization depends on D2R stimulation, it is tempting to speculate that repeated increases of phasic dopamine levels resulting from recurrent psychostimulant consumption by addict individuals could be responsible for the higher D2R GluN2B receptor proximity. The increased formation of D2R-GluN2B heteromerization we observed in human samples from psychostimulant abusers, together with interventional approaches in mice, emphasize their roles in the persistence of cocaine's behavioral effects. These important findings constitute a significant breakthrough in understanding of the molecular bases of cocaine-induced adaptations and highlight the potential benefit of targeting D2R-Glu2NB heteromerization, not only in the field of addiction, but also potentially for multiple neuropsychiatric disorders associated with an imbalance of DA and glutamate transmission.

\section{Materials and methods}

\section{Animals}

6-week-old C57BL/6J male mice were purchased from Janvier labs (Le Genest, St Isle, France). The animals were housed four per cage, in a 12-hour light-dark cycle, in stable temperature $\left(22^{\circ} \mathrm{C}\right)$ and humidity (60\%) conditions with ad libitum access to food and water. They were acclimatized to the animal facility for at least 1 week. All experiments were carried out in accordance with the standard ethical guidelines (European Community Council Directive on the Care and Use of Laboratory Animals (86/609/EEC) and the French National Committee (2010/63)).

\section{Drugs}

Drugs were administrated intraperitoneally in a volume of $10 \mathrm{ml} / \mathrm{kg}$. Cocaine hyd rochloride (Sigma Aldrich, St. Louis, MO) was dissolved in a saline solution $(0.9 \% \mathrm{NaCl}$ w/v).

9-tert-butyl doxycycline hydrochloride (9-TB-dox; Tebu-bio, Le Perray-en-Yvelines, France) was dissolved in a saline solution containing DMSO (5\%) and Tween20 (5\%). SCH23390 (0.25 mg/kg) or Eticlopride $(0.5 \mathrm{mg} / \mathrm{kg})$ dissolved in a saline solution $(0.9 \% \mathrm{NaCl}$ w/v) were administered $30 \mathrm{~min}$ prior to the challenge cocaine injection. 


\section{Viral constructions}

All AAV recombinant genomes were packaged in serotype 9 capsids. AAV-Tet-On-GluN1C1 expresses bicistronically the fluorescent reporter protein RFP and the $\mathrm{C} 1$ cassette of the GluN1 subunit (864DRKSGRAEPDPKKKATFRAITSTLASDT900) upon doxycycline (dox) treatment. The related control virus AAV-Tet-On-GluN1C1 $\Delta$ expresses a truncated version of $\mathrm{C} 1$ that is deleted from a stretch of 9 positively charged amino acids (890S890FKRRRSSK898), which are required for D1R-GluN1 interaction ${ }^{55}$. The AAV-Tet-On-D2R-IL3 encodes a sequence of the third intracellular loop of the D2R (225TKRSSRAFRA234) interacting with GluN2B. The control 9AAV-Tet-On-D2R-scr expresses a scrambled sequence (KFARRTSASR) of the D2R-IL3 (full AAV sequences are available upon request). All Tet-On AAV were injected bilaterally by infusing $0.7 \mu \mathrm{l}$ of a solution at $5.10^{13}$ viral genomes $/ \mathrm{ml}$ per hemisphere for the NAc ( $2 \mu \mathrm{l}$ for the dorsal striatum). The AAV-PPTA-Cre and AAV-PPE-Cre contain an expression cassette consisting of the Cre recombinase driven by the promoter of the PPTA gene (preprotachykinin) or the PPE gene (preproenkephalin), which are specifically expressed in D1R-MSN and D2RMSN, respectively $(7,39,40$ ) (see supplementary Fig. 3). AAV PPTA-cre or AAV-PPE-cre were coinjected with the AAV-pCAG-DIO-eGFP-WRPE (Upenn) expressing flexed eGFP under the CMV/actin hybrid promoter (CAG). All viruses were diluted in PBS pluronic $0.001 \%$.

\section{Stereotaxic injections}

Mice were anesthetized with ketamine $(150 \mathrm{mg} / \mathrm{kg})$ and xylazine $(10 \mathrm{mg} / \mathrm{kg})$ and placed on a stereotaxic apparatus (David Kopf Instruments, Tujunga, CA, USA). Craniotomies were realized using the following coordinates: $1.7 \mathrm{~mm}$ rostral to the bregma, $1.2 \mathrm{~mm}$ lateral to midline and $4.6 \mathrm{~mm}$ ventral to the skull surface to target the NAc and $1 \mathrm{~mm}$ rostral to the bregma, $1.8 \mathrm{~mm}$ lateral to midline and $3.25 \mathrm{~mm}$ ventral to the skull surface for the dorsal striatum. Viral injections were performed bilaterally at a rate of $0.15 \mu 1 / \mathrm{min}$ using a $10 \mu$ l-syringe (Hamilton 1700 series, Phymep, Paris, France) with a $200 \mu \mathrm{m}$ gauge needle (Phymep, Paris, France) mounted on a microinfusion pump (Harvard Apparatus, Holliston, MA). After the injection, the needle was left in place for an additional 8 min to avoid backflow.

\section{Doxycycline treatments}

Three weeks after stereotaxic injections of Tet-On AVV, the expression of the constructs was triggered by daily intraperitoneal (IP) injection of 9TB-dox (10 mg/kg) for 4 days (4d). To maintain expression mice were then supplemented with a mix containing doxycycline (dox) Hcl (2 mg/ml), 9TB-dox Hcl $(80 \mu \mathrm{g} / \mathrm{ml})$ and sucrose $(1 \%)$ added in drinking water. 


\section{Behavioral testing}

All behavioral tests were conducted during the light phase (8:00-19:00). Animals were randomly assigned to the saline or cocaine groups after viral injection. Prior to behavioral testing, mice were handled daily during $7 \mathrm{~d}$ in the experiment room. All mice were perfused with $4 \%(\mathrm{w} / \mathrm{v})$ paraformaldehyde (PFA) 24h post-behavior to systematically verify the accuracy of stereotaxic injections and expression of the RFP reporter protein. Mice that did not meet quality criterion (i.e. non-bilateral expression, off-target diffusion, excessive backflow or low RFP expression) were discarded from the study.

\section{Locomotor activity and cocaine psychomotor sensitization}

Locomotor activity was measured in a low luminosity environment inside a circular corridor (Immetronic, Pessac, France) containing four infrared beams placed at each $90^{\circ}$ angle. Locomotor activity was expressed as a cumulative count of crossings between quarters of the corridor for the indicated time. Mice were treated with dox $7 \mathrm{~d}$ before and until the end of the experiment. Mice were habituated to the test apparatus for $3 \mathrm{~d}$; basal locomotor activity was recorded on the third day of habituation. Cocaine sensitization experiments consisted of five daily 90 min sessions during which spontaneous activity was recorded for 30 min before saline or cocaine $(15 \mathrm{mg} / \mathrm{kg})$ injections and locomotor activity was then measured for 60 min postinjections. To study the consequences of uncoupling DAR from NMDAR on the maintenance of cocaineinduced locomotor sensitization, mice were treated for 5 consecutive days with saline or cocaine in the absence of dox. After the last injections, mice were supplemented with dox during a withdrawal period followed by a challenge injection of saline or cocaine.

\section{Cocaine Conditioned Place Preference (CPP)}

To study the impact of DAR-NMDAR heteromerization on the development of CPP, mice were treated with dox for $7 \mathrm{~d}$ before and until the end of the experiment. The CPP was performed in a two-compartment Plexiglas Y-maze apparatus (Imetronic). Each compartment contains different visual cues and floor textures for which mice did not show any preference on average before conditioning. All sessions lasted 20min. On day 1, mice were placed in the center of the apparatus and allowed to explore freely both compartments. Time spent in each compartment was automatically recorded. Mice spending more than $70 \%$ of the time in one compartment were excluded. On day 2, to avoid any initial preference bias, mice were randomly assigned to one or the other compartment for each group. Mice were injected with saline and placed immediately in the assigned closed compartment for $20 \mathrm{~min}$. After 1h, mice were injected with saline or cocaine and placed in the other closed compartment. This was repeated on day 3 . The test was performed 
on day 4, during which mice had a free access to both chambers. The CPP score was calculated as the difference between the time spent in the cocaine-paired chamber during day 4 minus the time spent in this compartment on day 1. CPP extinction and maintenance experiments were performed on the cocaine groups of mice injected with Tet-On-AVV that developed a preference for the cocaine-paired chamber in the absence of dox. Mice were then treated with dox until of the behavioral assessment. For the extinction phase, mice were injected with saline and put back in the apparatus with free access to both compartments for 20 min daily for 8 days. On the ninth day, mice were injected with cocaine and allowed to explore both compartments.

For palatable food-induced CPP, mice were food-deprived to $90 \%$ of initial ad libitum weight and treated with dox 7d prior and during behavioral assessment. Experiments were performed in the same apparatus and conditions as for cocaine-induced CPP with the following modifications: On day 2, after random group assignment. Mice were placed immediately in the assigned closed compartment containing chocolate crisps (Chocapic, Nestlé, Vevey, Switzerland) or nothing for $20 \mathrm{~min}$. After $1 \mathrm{~h}$, mice were placed in the other closed compartment. This was repeated on day 3 and 4 . The test was performed on day 5.

\section{Mouse tissue preparation}

Mice were anesthetized with an I.P injection of Euthasol (100 mg/kg; Le Vet, Oudewater, Netherland s) and perfused transcardially with $0.1 \mathrm{M} \mathrm{Na}_{2} \mathrm{HPO}_{4} / \mathrm{Na}_{2} \mathrm{HPO}_{4}, \mathrm{pH} 7.5$ containing $4 \%$ PFA at $4^{\circ} \mathrm{C}$. delivered with a peristaltic pump at $20 \mathrm{ml} / \mathrm{min}$ for $5 \mathrm{~min}$. Brains were then extracted, post-fixed overnight in $4 \%$ PFA, and stored at $4^{\circ} \mathrm{C} .30 \mu \mathrm{m}$-thick coronal sections were performed with a vibratome (Leica, Nussloch, Germany) and kept at $-20^{\circ} \mathrm{C}$ in a cryoprotective solution containing $30 \%$ ethylene glycol (v/v), 30\% glycerol (v/v) and 0.1 M PBS.

\section{Immunohistochemistry}

On day 1, free-floating sections were rinsed three times for $5 \mathrm{~min}$ in Tris-buffered saline (TBS, $0.9 \% \mathrm{NaCl}$, $0.1 \mathrm{M}$ Tris base, $\mathrm{pH}$ 7.5). Sections were then incubated in blocking solution containing $3 \%$ normal goat serum, $0.2 \%$ triton X-100 (Sigma, Aldrich) and $50 \mathrm{mM} \mathrm{NaF}$ for $2 \mathrm{~h}$ at room temperature (RT) before overnight $4^{\circ} \mathrm{C}$ incubation with primary antibodies (see antibody table) diluted in the blocking buffer. On day 2, after three 10-min rinses in TBS, sections were incubated for 90 min with secondary antibodies (see antibody table). The anti-FosB antibody recognizes full-length FosB as well as $\Delta$ FosB but at the time point studied (24h post cocaine) all FosB-immunoreactive protein represents $\Delta$ FosB (57). Afterthree 5-min rinses in TBS, sections were incubated for 5 min with Hoechst (Invitrogen) for nuclei counterstaining. Three 5- 
min TBS and two Tris buffer (0.1 M Tris base, $\mathrm{pH} 7.5)$ washes were performed before sections were mounted in Prolong Gold (Invitrogen).

\section{Immunoblotting}

Mice were killed by decapitation and their heads were immediately snap frozen in liquid nitrogen. Microdiscs of NAc were punched out using disposable biopsy punches (1 mm diameter) (Kai medical) and stored in individual tubes at $-80^{\circ} \mathrm{C}$. Microdiscs were then homogenized by sonication in a lysis solution containing $50 \mathrm{mM}$ Tris $\mathrm{Hcl}, 2 \% \mathrm{SDS}, 0.5 \mathrm{M}$ urea diluted in water. Protein concentrations were determined using the BCA assay kit (Pierce, Rockford, IL). A fixed amount of protein (30 $\mu \mathrm{g}$ per lane) was separated by SDS-polyacrylamide gel electrophoresis (12\%) before electrophoretic transfer onto nitrocellulose membranes. Membranes were incubated $1 \mathrm{~h}$ at RT in a blocking solution containing TBS with $0.1 \%$ tween and 5\% non-fat dry milk. Membranes were incubated at $4{ }^{\circ} \mathrm{C}$ overnight with primary antibodies (see antibody table). Membranes were then washed three times in TBS-Tween and then incubated for $1 \mathrm{~h}$ at RT with secondary antibodies (see antibody table) coupled to horseradish peroxidase (HRP). Immunoreactive bands were detected by chemoluminescent detection (ECL kit, GE Healthcare) and images were acquired using the ImageQuant LAS 4000 (GE Healthcare Life Science). The densitometry of immunoreactive bands was quantified using ImageJ and normalized to the loading control.

\section{Human brain samples}

Brain samples from individuals with a history of substance dependence, with toxicological evidence of current psychostimulant use, and from matched healthy controls ( $\mathrm{n}=13$ /group; Table $\mathrm{S} 1)$ were provided by the Suicide section of the Douglas-Bell Canada Brain Bank (DBCBB). Brains were donated to the DBCBB by familial consent through the Quebec Coroner's Office, which ascertained the cause of death. Two months after death, psychological autopsies with next-of-kin were conducted, as previously described (58).

Dissections of ventral striatum were performed with the guidance of a human brain atlas (59) on $0.5 \mathrm{~cm}$ thick formalin-fixed coronal brain sections at an anatomical level equivalent to plate 15 of this atlas (-7.5 $\mathrm{mm}$ from the center of the anterior commissure). Tissue was extracted rostral to the anterior commissure and ventral to the tip of the anterior limb of internal capsule and fixed by immersion in formalin until paraffin embedding. The latter was completed using a Leica ASP200S automated processor. Tissue blocks were dehydrated in increasing gradients of alcohol $(70 \%, 95 \%, 3 \times 100 \%)$ for $1.5 \mathrm{hr}$ each, followed by clearing in 3 changes of $100 \%$ xylene ( $2 \mathrm{hr}, 2 \times 1.5 \mathrm{hr}$ ). The samples were then infiltrated in 3 changes molten 
paraffin, 3hrs each before embedding. Slices of $6 \mu \mathrm{m}$ thickness when then prepared with microtome at the histology facility of the ICM institute (Paris).

\section{Proximity Ligation Assay}

Proximity ligation assay (PLA) on mouse brain sections (30 $\mu \mathrm{m}$-thick) was performed in 48 -well plates according to the manufacturer's instructions for free-floating sections. PLA on human post-mortem paraffin-embedded tissue was performed on sections mounted on superfrost plus slides (Thermo Scientific). $6 \mu$ m-thick human caudate-putamen sections were deparaffinized in xylene, rehydrated in graded ethanol series and washed briefly in TBS. For antigen retrieval, sections were boiled for 6 min in sodium citrate buffer (10 mM, pH 6). For brightfield PLA, sections were incubated for $30 \mathrm{~min}$ at RT in TBS containing $1 \% \mathrm{H}_{2} \mathrm{O}_{2}$ to block endogenous peroxidase. After three 5 min rinses with TBS containing $0.1 \%$ triton X-100 (TBS-T), sections were incubated 1h at RT with blocking buffer (Duolink blocking buffer for PLA) then with primary antibodies (see antibody table) diluted in the Duolink antibody dilution buffer overnight at $4^{\circ} \mathrm{C}$. Anti-rat PLA plus and minus probes were made using the PLA probemaker kit (Sigma Aldrich) with a Goat anti-rat IgG antibody (Jackson Immunoresearch) according to manufacturer's instructions. Immunofluorescent PLA and the remaining procedures for brightfield PLA were performed as previously described (31). For brightfield PLA nuclei were counterstained using the duolink nuclear stain. In this study, we used both single-recognition PLA, using only one primary antibody to detect single antigen and dual-recognition to detect DAR-NMDAR complexes using two primary antibodies.

\section{Antibodies}

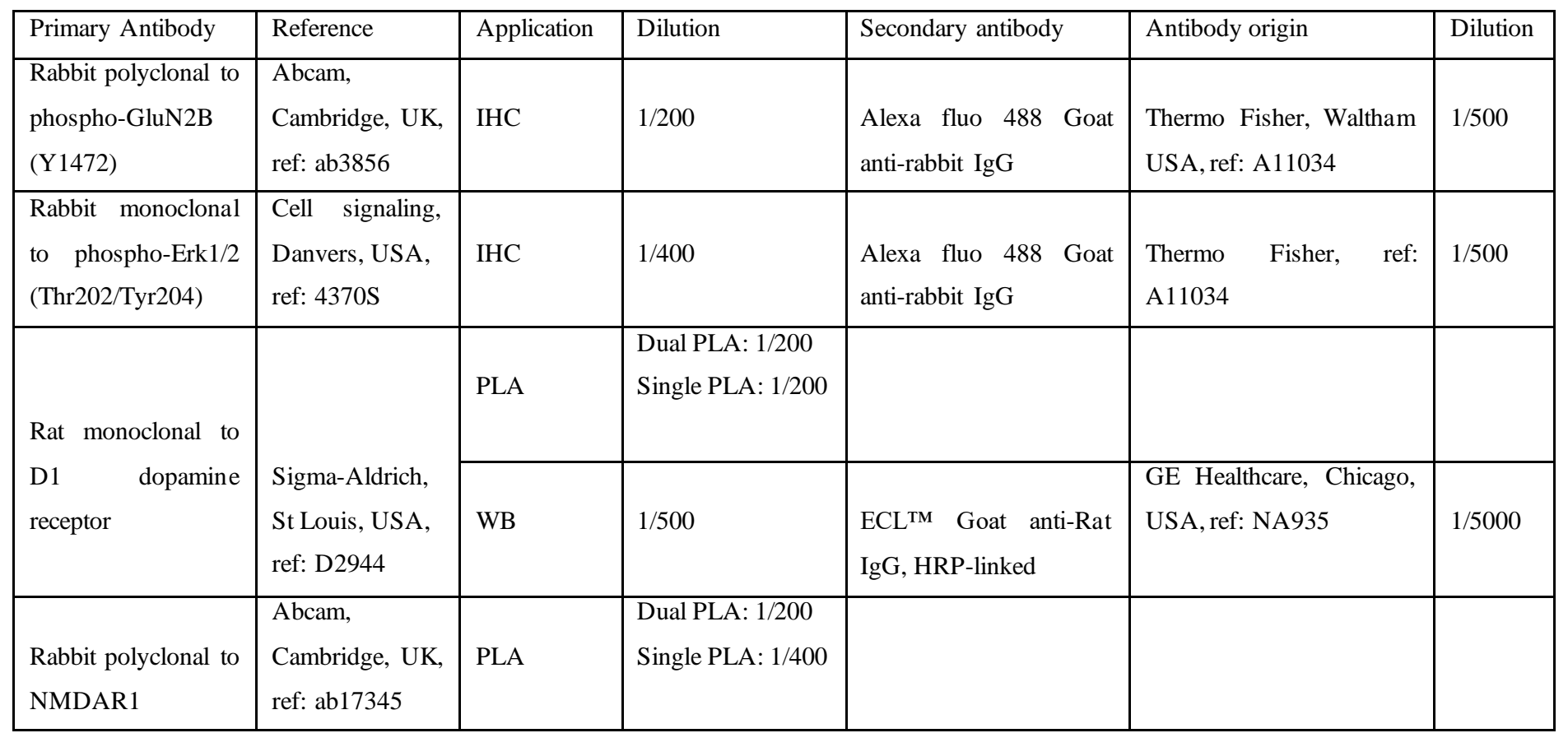




\begin{tabular}{|c|c|c|c|c|c|c|}
\hline \multirow{2}{*}{$\begin{array}{l}\text { Rabbit polyclonal to } \\
\text { D2 dopamine } \\
\text { receptor }\end{array}$} & \multirow{2}{*}{$\begin{array}{l}\text { Millipore, } \\
\text { Burlington, } \\
\text { USA } \\
\text { ABN462 }\end{array}$} & PLA & $\begin{array}{l}\text { Dual PLA: } 1 / 200 \\
\text { Single PLA: } 1 / 400\end{array}$ & & & \\
\hline & & WB & $1 / 500$ & $\begin{array}{l}\text { WB: ECL }{ }^{\mathrm{TM}} \text { Goat anti- } \\
\text { Rabbit IgG, HRP-linked }\end{array}$ & $\begin{array}{l}\text { GE Healthcare, } \\
\text { ref: NA934 }\end{array}$ & $1 / 5000$ \\
\hline & & PLA & $\begin{array}{l}\text { Dual PLA: } 1 / 200 \\
\text { Single PLA: } 1 / 500\end{array}$ & & & \\
\hline $\begin{array}{l}\text { Mouse monoclonal } \\
\text { to NMDAR2B NT }\end{array}$ & $\begin{array}{l}\text { Millipore, } \\
\text { ref: MAB5782 }\end{array}$ & WB & $1 / 500$ & $\begin{array}{l}\text { WB: ECL }{ }^{\mathrm{TM}} \text { Goat anti- } \\
\text { Mouse IgG, HRP-linked }\end{array}$ & $\begin{array}{l}\text { GE Healthcare, Chicago, } \\
\text { USA, ref: NA931 }\end{array}$ & $1 / 5000$ \\
\hline $\begin{array}{l}\text { Mouse Monoclonal } \\
\text { to NMDAR1 CT }\end{array}$ & $\begin{array}{l}\text { Millipore, } \\
\text { ref: } 05-432\end{array}$ & WB & WB: $1 / 200$ & $\begin{array}{l}\text { WB: ECL }{ }^{\mathrm{TM}} \text { Goat anti- } \\
\text { Mouse IgG, HRP-linked }\end{array}$ & $\begin{array}{l}\text { GE Healthcare, } \\
\text { ref: NA931 }\end{array}$ & $1 / 5000$ \\
\hline $\begin{array}{l}\text { Rabbit Polyclonal to } \\
\text { Fos B }\end{array}$ & $\begin{array}{l}\text { Santa Cruz, } \\
\text { Ref: sc-48 }\end{array}$ & IHC & $1 / 500$ & Cy5 goat anti-rabbit $\operatorname{IgG}$ & $\begin{array}{l}\text { Jackson ImmunoResearch } \\
\text { Ref: AB_2338013 }\end{array}$ & $1 / 1000$ \\
\hline
\end{tabular}

\section{Image acquisition and analysis}

For immunochemistry and fluorescent PLA staining, images were taken with a confocal laser scanning microscope (SP5, Leica) using a 20X and a 63X objective (oil immersion, Leica) respectively. The pinhole was set to 1 Airy unit, excitation wavelength and emission range were 495 and 500-550 nm for green PLA signal, 488 and 500-550 nm for Alexa fluo 488 and 590-650 nm for RFP. Laser intensity and detector gain were constant for all image acquisitions. Images were acquired in a range of $5 \mu \mathrm{m}$ with a z-step of $0.2 \mu \mathrm{m}$. Conditions were run in duplicate and quantifications were made from at least 4 images per condition. Four mice were used for each condition. Maximum projection images were analyzed using ImageJ (National Institutes of Health, Bethesda, MD).

For PLA images quantification, ImageJ was used to construct a mask from RFP positive cells. The mask was then fused with the PLA signal-containing channel using the image multiply function. The PLA punctate signal was quantified on the resulting image in ICY using the spot detector function (detector $=$ scale 3: 65; filtering = Min size: 6; Max size: 30); these parameters were chosen manually from random images to obtain optimal signal-to-noise ratio and minimal false positive in images from negative control conditions. RFP-positive cells showing at least one PLA puncta for one or the other heteromer subtypes were included as this allowed us to identify the MSN subtype analyzed (D1R-MSN v.s D2R-MSN). RFP positive cells which did not present any D1R-GluN1 or D2R-GluN2B PLA signal were discarded.

Mouse brightfield PLA images were taken using a microscope (Leica, DM4000) with a 63X objective. A minimum of 6 rand om fields per structure per mice were taken. 
For human brightfield PLA, whole-slide images were taken using a Zeiss, Axioscan with a 40X magnification and z-stack with a $1 \mu \mathrm{m}$ step interval. Whole-slide scanned images were visualized with ZEN Blue edition lite software (Zeiss, version 3.1). 25 region of interest (ROI) of $500 \mu \mathrm{m} \times 500 \mu \mathrm{m}$ were generated randomly in the NAc and were exported as TIFF files using the image export plugin. The z-stack with the maximum number of PLA signal in the focus was chosen for each image.

Human and mouse brightfield PLA Images were analyzed using an ImageJ homemade macro that use the Find Maxima tool to detect the PLA punctate with prominence set at $>40$. Results are represented as the mean PLA signal density per field of views.

For quantification of immunohistochemistry experiments, immunoreactive cells, were analyzed using ImageJ considering the cells with immunofluorescence above a fixed threshold.

\section{Neuronal survival}

Neuronal survival was quantified manually based on Hoescht-counterstained nuclei. Survival was defined as the percentage of viable neurons exhibiting large, uniform nuclei and even distribution of Hoescht among RFP positive cells over RFP positive cells classified as apoptotic based on, at least, two of the following criteria: condensed nuclei, single chromatin clump, small nuclei size, non-circular nuclei shape and increased Hoescht intensity.

\section{Spine density analysis}

Twenty-four hours after the CPP paradigm, mice were perfused and brains were sliced as described above. Dendrite spine analysis was performed on RFP-positive dendrites from D1R-MSN and D2R-MSN identified based on the presence of GFP driven by the co-injection of AAV-PPTA-cre/AAV-pCAG-DIOeGFP or AAV-PPE-cre/pCAG-DIO-eGFP, respectively. Image stacks were taken using a confocal laser scanning microscope (SP5, Leica). For analysis of D1R-MSN, images were collected through 63x objective with pixel size of $65 \mathrm{~nm}$ and z-step of $200 \mathrm{~nm}$. For D2R-MSN, images were collected through 40x objective with pixel size of $95 \mathrm{~nm}$ and z-step of $300 \mathrm{~nm}$. The excitation wavelength was 488 for GFP and 561 for RFP, with emission range 500-550 nm and 570-650 nm, respectively. Images were acquired in sequential mode with a Hybrid detector (HyD, Leica). Deconvolution with experimental point spread function from fluorescent beads using a maximum likelihood estimation algorithm was performed with Huygens software (Scientific Volume Imaging). Neuronstudio software was used to reconstruct the dendrite and detect dendritic spines with manual correction.

\section{Patch clamp recordings.}


Mice were anesthetized (Ketamine $150 \mathrm{mg} / \mathrm{kg} /$ Xylazine $10 \mathrm{mg} / \mathrm{kg}$ ) and transcardially perfused with aCSF for slice preparation. Coronal $250 \mu \mathrm{m}$ slices containing the nucleus accumbens were obtained in bubbled ice-cold $95 \% \mathrm{O}_{2} / 5 \% \mathrm{CO}_{2}$ aCSF containing (in $\mathrm{mM}$ ): $\mathrm{KCl} 2.5, \mathrm{NaH}_{2} \mathrm{PO}_{4} 1.25, \mathrm{MgSO}_{4} 10, \mathrm{CaCl}_{2} 0.5$, glucose 11, sucrose 234, $\mathrm{NaHCO}_{3} 26$, using a HM650V vibratome (Microm, France). Slices were then incubated in aCSF containing (in mM): $\mathrm{NaCl} 119, \mathrm{KCl} 2.5, \mathrm{NaH}_{2} \mathrm{PO}_{4} 1.25, \mathrm{MgSO}_{4} 1.3, \mathrm{CaCl}_{2} 2.5, \mathrm{NaHCO}_{3} 26$, glucose 11 , at $37^{\circ} \mathrm{C}$ for $1 \mathrm{~h}$, and then kept at room temperature. Slices were transferred and kept at $31^{\circ} \mathrm{C}$ in a recording chamber superfused with $2 \mathrm{ml} / \mathrm{min}$ aCSF in the continuous presence of $50 \mu \mathrm{M}$ picrotoxin (SigmaAldrich, France, dissolved in DMSO) to block GABAergic transmission. Neurons were visualized by combined epifluorescent and infrared/differential interference contrast visualization using an Olympus upright microscope holding 5x and 40x objectives. Whole-cell voltage-clamp recording techniques were used to measure synaptic responses using a Multiclamp 700B (Molecular Devices, Sunnyvale, CA). Signals were collected and stored using a Digidata 1440A converter and pCLAMP 10.2 software (Molecular Devices, CA). AMPA-R/NMDA-R ratio was assessed using an internal solution containing (in $\mathrm{mM}$ ) 130 $\mathrm{CsCl}, 4 \mathrm{NaCl}, 2 \mathrm{MgCl}_{2}$, 1.1 EGTA, 5 HEPES, $2 \mathrm{Na}$ ATP, 5 sodium creatine phosphate, $0.6 \mathrm{Na} 3 \mathrm{GTP}$ and 0.1 spermine. Synaptic currents were evoked by stimuli $(60 \mu \mathrm{s})$ at $0.1 \mathrm{~Hz}$ through a glass pipette placed 200 $\mu \mathrm{m}$ from the patched neurons. Evoked-EPSCs were obtained at $\mathrm{V}=+40 \mathrm{mV}$ in the absence and presence of the AMPA-R antagonist DNQX. In all cases, 30 consecutive EPSCs were averaged and offline analyses was performed using Clampfit 10.2 (Axon Instruments, USA) and Prism (Graphpad, USA). Pharmacologically isolated EPSC NMDAR decay time, recorded from cells voltage clamped at $+40 \mathrm{mV}$, was fitted with a double exponential function, using Clampfit software, to calculate both slow and fast decay time constants, $\tau$ fast and $\tau$ slow, respectively. The weighted time constant ( $\tau$ weighted) was calculated using the relative contribution from each of these components, applying the formula: $\tau \mathrm{w}=[(\mathrm{af} . \tau \mathrm{f})+($ as. $\tau \mathrm{s})] /(\mathrm{af}+$ as), where af and as are the relative amplitudes of the two exponential components, and $\tau \mathrm{f}$ and $\tau \mathrm{s}$ are the corresponding time constants.

\section{Cyclic AMP accumulation assay}

HEK 293 cells stably expressing the D2R (60) were grown on polylysine (Poly-D-lysine hydrobromide, Sigma) and transfected with Tet-On plasmids encoding either the D2R-IL3 or D2R-IL3-scr peptides and the Tag RFP (4 $\mu$ g per well), complexed with Lipofectamine ${ }^{\circledR} 2000$ transfection reagent (Invitrogen ${ }^{\mathrm{TM}}$ ), in DMEM (DMEM high glucose GlutaMAX ${ }^{\mathrm{TM}}$ Supplement pyruvate, Gibco), and incubated for 5 h. Posttransfection, cells were rinsed and wells filled with DMEM supplemented with $10 \% \mathrm{FBS}$ and antibiotics to induce D2R expression (Hygromycine $2 \mu \mathrm{l} / \mathrm{ml}$ (Sigma Aldrich); Blasticidine 1,5 $\mu \mathrm{l} / \mathrm{ml}$ (Cayla Invivogen ant-bl-1); Tetracycline (Sigma T7660-5g) $1 \mu \mathrm{l} / \mathrm{ml}$ ) and incubated overnight. Peptide expression was 
induced by addition of $1 \mu \mathrm{g} / \mathrm{ml}$ dox solution, incubating for 48 hours. Following the dox treatment, cells were rinsed in DMEM before pretreatment with 1mM IsoButylMethylXanthine (IBMX, Sigma Aldrich) for $15 \mathrm{~min}$. Cells were then stimulated for $30 \mathrm{~min}$ with the indicated concentrations of agonist Quinpirole (Tocris), in the presence of $1 \mathrm{mM}$ IBMX and $10 \mu \mathrm{M}$ Forskolin (Tocris). Endogenous phosphodiesterase activity was stopped by aspiration of the medium and the addition of $0.1 \mathrm{M} \mathrm{HCL}(300 \mu \mathrm{l} /$ well). After centrifugation at $600 \mathrm{~g}$ during $10 \mathrm{~min}$, protein concentration of supernatants was quantified by BCA (Uptima, Interchim). Cyclic AMP levels were determined in samples containing $10 \mu \mathrm{g}$ of protein. The accumulation of cAMP was measured by using a cAMP Enzyme Immunoassay kit (Sigma Aldrich) as described by the manufacturer using Victor3 (Perkin Elmer) plate reader. The curve fit was obtained by GraphPad Prism 8 (GraphPad Software, Inc.).

\section{Statistical analysis}

Results were analyzed with Graphpad Prism (version 8.0.1). Sample size was predetermined on the basis of published studies, pilot experiments and in-house expertise. All data are displayed as mean +/- SEM. Two-tailed Student's test was used for the comparison of two ind ependent groups. For more than two groups comparison, one-way, two-way or three-way repeated-measures ANOVA were performed followed by Bonferoni Post-hoc test. Data distribution was assumed to be normal and variances were assumed to be homogenous. The main effect and post-hoc statistical significances are given in the appropriate figure legend for each experiment.

\section{Data availability}

The datasets that support the findings of this study are available from the corresponding author upon reasonable request.

\section{References}

1. C. Lüscher, R. C. Malenka, Drug-Evoked Synaptic Plasticity in Addiction: From Molecular Changes to Circuit Remodeling. Neuron. 69, 650-663 (2011).

2. M. Salery, P. Trifilieff, J. Caboche, P. Vanhoutte, From Signaling Molecules to Circuits and Behaviors: Cell-Type-Specific Adaptations to Psychostimulant Exposure in the Striatum. Biological Psychiatry. 87, 944-953 (2020).

3. N. D. Volkow, M. Morales, The Brain on Drugs: From Reward to Addiction. Cell. 162, 712-725 (2015). 
4. S. E. Hyman, R. C. Malenka, E. J. Nestler, NEURAL MECHANISMS OF ADDICTION: The Role of Reward-Related Learning and Memory. Annual Review of Neuroscience. 29, 565-598 (2006).

5. J. Bertran-Gonzalez, C. Bosch, M. Maroteaux, M. Matamales, D. Herve, E. Valjent, J.-A. Girault, Opposing Patterns of Signaling Activation in Dopamine D1 and D2 Receptor-Expressing Striatal Neurons in Response to Cocaine and Haloperidol. Journal of Neuroscience. 28, 5671-5685 (2008).

6. S. M. Ferguson, D. Eskenazi, M. Ishikawa, M. J. Wanat, P. E. M. Phillips, Y. Dong, B. L. Roth, J. F. Neumaier, Transient neuronal inhibition reveals opposing roles of indirect and direct pathways in sensitization. Nature Neuroscience. 14, 22-24 (2011).

7. T. Hikida, K. Kimura, N. Wada, K. Funabiki, S. Nakanishi, Distinct Roles of Synaptic Transmission in Direct and Indirect Striatal Pathways to Reward and Aversive Behavior. Neuron. 66, 896-907 (2010).

8. A. V. Kravitz, L. D. Tye, A. C. Kreitzer, Distinct roles for direct and indirect pathway striatal neurons in reinforcement. Nature Neuroscience. 15, 816-818 (2012).

9. M. K. Lobo, H. E. Covington, D. Chaudhury, A. K. Friedman, H. Sun, D. Damez-Werno, D. M. Dietz, S. Zaman, J. W. Koo, P. J. Kennedy, E. Mouzon, M. Mogri, R. L. Neve, K. Deisseroth, M.-H. Han, E. J. Nestler, Cell Type-Specific Loss of BDNF Signaling Mimics Optogenetic Control of Cocaine Reward. Science. 330, 385-390 (2010).

10. R. Bock, J. H. Shin, A. R. Kaplan, A. Dobi, E. Markey, P. F. Kramer, C. M. Gremel, C. H. Christensen, M. F. Adrover, V. A. Alvarez, Strengthening the accumbal indirect pathway promotes resilience to compulsive cocaine use. Nature Neuroscience. 16, 632-638 (2013).

11. P. F. Durieux, B. Bearzatto, S. Guiducci, T. Buch, A. Waisman, M. Zoli, S. N. Schiffmann, A. de K. d'Exaerde, D 2 R striatopallidal neurons inhibit both locomotor and drug reward processes. Nat Neurosci. 12, 393-395 (2009).

12. P. F. Durieux, S. N. Schiffmann, A. de Kerchove d'Exaerde, Differential regulation of motor control and response to dopaminergic drugs by D1R and D2R neurons in distinct dorsal striatum subregions: Dorsal striatum D1R- and D2R-neuron motor functions. The EMBO Journal. 31, 640-653 (2012).

13. V. Pascoli, M. Turiault, C. Lüscher, Reversal of cocaine-evoked synaptic potentiation resets druginduced adaptive behaviour. Nature. 481, 71-75 (2012).

14. V. Pascoli, J. Terrier, J. Espallergues, E. Valjent, E. C. O’Connor, C. Lüscher, Contrasting forms of cocaine-evoked plasticity control components of relapse. Nature. 509, 459-464 (2014).

15. V. Pascoli, A. Hiver, R. V. Zessen, M. Loureiro, R. Achargui, M. Harada, J. Flakowski, C. Lüscher, Stochastic synaptic plasticity underlying compulsion in a model of addiction. Nature. 564, 366-371 (2018).

16. J. Terrier, C. Lüscher, V. Pascoli, Cell-Type Specific Insertion of GluA2-Lacking AMPARs with Cocaine Exposure Leading to Sensitization, Cue-Induced Seeking and Incubation of Craving. Neuropsychopharmacology. 41, 1779-1789 (2016). 
17. C. L. Heusner, R. D. Palmiter, Expression of Mutant NMDA Receptors in Dopamine D1 ReceptorContaining Cells Prevents Cocaine Sensitization and Decreases Cocaine Preference. J. Neurosci. 25, 6651-6657 (2005).

18. J. Schumann, R. Yaka, Prolonged Withdrawal from Repeated Noncontingent Cocaine Exposure Increases NMDA Receptor Expression and ERK Activity in the Nucleus Accumbens. J. Neurosci. 29, 6955-6963 (2009).

19. L. R. Beutler, M. J. Wanat, A. Quintana, E. Sanz, N. S. Bamford, L. S. Zweifel, R. D. Palmiter, Balanced NMDA receptor activity in dopamine D1 receptor (D1R)- and D2R-expressing medium spiny neurons is required for amphetamine sensitization. Proceedings of the National Academy of Sciences. 108, 4206-4211 (2011).

20. V. Pascoli, A. Besnard, D. Hervé, C. Pagès, N. Heck, J.-A. Girault, J. Caboche, P. Vanhoutte, Cyclic Adenosine Monophosphate-Independent Tyrosine Phosphorylation of NR2B Mediates CocaineInduced Extracellular Signal-Regulated Kinase Activation. Biological Psychiatry. 69, 218-227 (2011).

21. V. Pascoli, E. Cahill, F. Bellivier, J. Caboche, P. Vanhoutte, Extracellular Signal-Regulated Protein Kinases 1 and 2 Activation by Addictive Drugs: A Signal Toward Pathological Adaptation. Biological Psychiatry. 76, 917-926 (2014).

22. M. E. Joffe, S. R. Vitter, B. A. Grueter, GluN1 deletions in D1- and A2A-expressing cell types reveal distinct modes of behavioral regulation. Neuropharmacology. 112, 172-180 (2017).

23. A. Andrianarivelo, E. Saint-Jour, R. Walle, P. Trifilieff, P. Vanhoutte, Modulation and functions of dopamine receptor heteromers in drugs of abuse-induced adaptations. Neuropharmacology. 152, 4250 (2019).

24. D. O. Borroto-Escuela, J. Carlsson, P. Ambrogini, M. Narváez, K. Wydra, A. O. Tarakanov, X. Li, C. Millón, L. Ferraro, R. Cuppini, S. Tanganelli, F. Liu, M. Filip, Z. Diaz-Cabiale, K. Fuxe, Understanding the Role of GPCR Heteroreceptor Complexes in Modulating the Brain Networks in Health and Disease. Front. Cell. Neurosci. 11 (2017), doi:10.3389/fncel.2017.00037.

25. S. Ferré, C. Quiroz, M. Orru, X. Guitart, G. Navarro, A. Cortés, V. Casadó, E. I. Canela, C. Lluis, R. Franco, Adenosine A(2A) Receptors and A(2A) Receptor Heteromers as Key Players in Striatal Function. Front Neuroanat. 5, 36 (2011).

26. K. Fuxe, D. O. Borroto-Escuela, W. Romero-Fernandez, M. Palkovits, A. O. Tarakanov, F. Ciruela, L. F. Agnati, Moonlighting Proteins and Protein-Protein Interactions as Neurotherapeutic Targets in the G Protein-Coupled Receptor Field. Neuropsychopharmacology. 39, 131-155 (2014).

27. M. Wang, A. H. Wong, F. Liu, Interactions between NMDA and dopamine receptors: A potential therapeutic target. Brain Research. 1476, 154-163 (2012).

28. F. J. S. Lee, S. Xue, L. Pei, B. Vukusic, N. Chéry, Y. Wang, Y. T. Wang, H. B. Niznik, X. Yu, F. Liu, Dual Regulation of NMDA Receptor Functions by Direct Protein-Protein Interactions with the Dopamine D1 Receptor. Cell. 111, 219-230 (2002).

29. L. Pei, F. J. S. Lee, A. Moszczynska, B. Vukusic, F. Liu, Regulation of Dopamine D1 Receptor Function by Physical Interaction with the NMDA Receptors. J. Neurosci. 24, 1149-1158 (2004). 
30. C. Fiorentini, F. Gardoni, P. Spano, M. D. Luca, C. Missale, Regulation of Dopamine D1 Receptor Trafficking and Desensitization by Oligomerization with Glutamate N-Methyl-D-aspartate Receptors. J. Biol. Chem. 278, 20196-20202 (2003).

31. E. Cahill, V. Pascoli, P. Trifilieff, D. Savoldi, V. Kappès, C. Lüscher, J. Caboche, P. Vanhoutte, D1R/GluN1 complexes in the striatum integrate dopamine and glutamate signalling to control synaptic plasticity and cocaine-induced responses. Mol Psychiatry. 19, 1295-1304 (2014).

32. X.-Y.Liu, X.-P. Chu, L.-M. Mao, M. Wang, H.-X. Lan, M.-H. Li, G.-C. Zhang, N. K. Parelkar, E. E. Fibuch, M. Haines, K. A. Neve, F. Liu, Z.-G. Xiong, J. Q. Wang, Modulation of D2R-NR2B Interactions in Response to Cocaine. Neuron. 52, 897-909 (2006).

33. T. E. Robinson, K. C. Berridge, The neural basis of drug craving: An incentive-sensitization theory of addiction. Brain Research Reviews. 18, 247-291 (1993).

34. P. Trifilieff, M.-L. Rives, E. Urizar, R. Piskorowski, H. Vishwasrao, J. Castrillon, C. Schmauss, M. Slättman, M. Gullberg, J. Javitch, Detection of antigen interactions ex vivo by proximity ligation assay: endogenous dopamine D2-adenosine A2A receptor complexes in the striatum. BioTechniques. 51, 111-118 (2011).

35. A. L. Frederick, H. Yano, P. Trifilieff, H. D. Vishwasrao, D. Biezonski, J. Mészáros, E. Urizar, D. R. Sibley, C. Kellendonk, K. C. Sonntag, D. L. Graham, R. J. Colbran, G. D. Stanwood, J. A. Javitch, Evidence against dopamine D1/D2 receptor heteromers. Molecular Psychiatry. 20, 1373-1385 (2015).

36. W.-D. Yao, R. R. Gainetdinov, M. I. Arbuckle, T. D. Sotnikova, M. Cyr, J.-M. Beaulieu, G. E. Torres, S. G. N. Grant, M. G. Caron, Identification of PSD-95 as a Regulator of Dopamine-Mediated Synaptic and Behavioral Plasticity. Neuron. 41, 625-638 (2004).

37. J. Zhang, T.-X. Xu, P. J. Hallett, M. Watanabe, S. G. N. Grant, O. Isacson, W.-D. Yao, PSD-95 Uncouples Dopamine-Glutamate Interaction in the D1/PSD-95/NMDA Receptor Complex. Journal of Neuroscience. 29, 2948-2960 (2009).

38. A. S. Woods, F. Ciruela, K. Fuxe, L. F. Agnati, C. Lluis, R. Franco, S. Ferré, Role of electrostatic interaction in receptor-receptor heteromerization. J Mol Neurosci. 26, 125-132 (2005).

39. M. Dos Santos, M. Salery, B. Forget, M. A. Garcia Perez, S. Betuing, T. Boudier, P. Vanhoutte, J. Caboche, N. Heck, Rapid Synaptogenesis in the Nucleus Accumbens Is Induced by a Single Cocaine Administration and Stabilized by Mitogen-Activated Protein Kinase Interacting Kinase-1 Activity. Biological Psychiatry. 82, 806-818 (2017).

40. S. Yagishita, A. Hayashi-Takagi, G. C. R. Ellis-Davies, H. Urakubo, S. Ishii, H. Kasai, A critical time wind ow for dopamine actions on the structural plasticity of dendritic spines. Science. 345, 1616-1620 (2014).

41. J. Kim, B.-H. Park, J. H. Lee, S. K. Park, J.-H. Kim, Cell Type-Specific Alterations in the Nucleus Accumbens by Repeated Exposures to Cocaine. Biological Psychiatry. 69, 1026-1034 (2011).

42. Y. Zhu, J. Mészáros, R. Walle, R. Fan, Z. Sun, A. J. Dwork, P. Trifilieff, J. A. Javitch, Detecting G protein-coupled receptor complexes in postmortem human brain with proximity ligation assay and a Bayesian classifier. BioTechniques. 68, 122-129 (2019). 
43. Y.Zhu, A. J. Dwork, P. Trifilieff, J. A. Javitch, Detection of G Protein-Coupled Receptor Complexes in Postmortem Human Brain by Proximity Ligation Assay. Current Protocols in Neuroscience. 91, e86 (2020).

44. G. F. Koob, N. D. Volkow, Neurobiology of addiction: a neurocircuitry analysis. The Lancet Psychiatry. 3, 760-773 (2016).

45. P. Trifilieff, F. Ducrocq, S. van der Veldt, D. Martinez, Blunted Dopamine Transmission in Addiction: Potential Mechanisms and Implications for Behavior. Seminars in Nuclear Medicine. 47, 64-74 (2017).

46. N. D. Volkow, J. S. Fowler, A. P. Wolf, D. Schlyer, C. Y. Shiue, R. Alpert, S. L. Dewey, J. Logan, B. Bendriem, D. Christman, Effects of chronic cocaine abuse on postsynaptic dopamine receptors. Am J Psychiatry. 147, 719-724 (1990).

47. N. D. Volkow, G.-J. Wang, J. S. Fowler, J. Logan, S. J. Gatley, A. Gifford, R. Hitzemann, Y.-S. Ding, N. Pappas, Prediction of Reinforcing Responses to Psychostimulants in Humans by Brain Dopamine D2 Receptor Levels. AJP. 156, 1440-1443 (1999).

48. N. D. Volkow, G.-J. Wang, J. S. Fowler, D. Tomasi, F. Telang, Addiction: Beyond dopamine reward circuitry. Proceedings of the National Academy of Sciences. 108, 15037-15042 (2011).

49. M. Creed, N. R. Ntamati, R. Chandra, M. K. Lobo, C. Lüscher, Convergence of Reinforcing and Anhedonic Cocaine Effects in the Ventral Pallidum. Neuron. 92, 214-226 (2016).

50. A. F. MacAskill, J. M. Cassel, A. G. Carter, Cocaine exposure reorganizes cell type-and input-specific connectivity in the nucleus accumbens. Nature Neuroscience. 17, 1198-1207 (2014).

51. M. K. Lobo, E. J. Nestler, The Striatal Balancing Act in Drug Addiction: Distinct Roles of Direct and Indirect Pathway Medium Spiny Neurons. Frontiers in Neuroanatomy. 5 (2011), doi:10.3389/fnana.2011.00041.

52. P. Sun, J. Wang, W. Gu, W. Cheng, G. Jin, E. Friedman, J. Zheng, X. Zhen, PSD-95 regulates D 1 dopamine receptor resensitization, but not receptor-mediated Gs-protein activation. Cell Res. 19, 612624 (2009).

53. P. Paoletti, C. Bellone, Q. Zhou, NMDA receptor subunit diversity: impact on receptor properties, synaptic plasticity and disease. Nat Rev Neurosci. 14, 383-400 (2013).

54. P. W. Kalivas, K. McFarland, Brain circuitry and the reinstatement of cocaine-seeking behavior. Psychopharmacology (Berl). 168, 44-56 (2003).

55. S. S. Song, B. J. Kang, L. Wen, H. J. Lee, H. Sim, T. H. Kim, S. Yoon, B.-J. Yoon, G. J. Augustine, J.-H. Baik, Optogenetics reveals a role for accumbal medium spiny neurons expressing dopamine D2 receptors in cocaine-induced behavioral sensitization. Front. Behav. Neurosci. 8 (2014), doi:10.3389/fnbeh.2014.00336.

56. D. Belin, A. Belin-Rauscent, J. E. Murray, B. J. Everitt, Addiction: failure of control over maladaptive incentive habits. Current Opinion in Neurobiology. 23, 564-572 (2013). 
57. M. K. Lobo, S. Zaman, D. M. Damez-Werno, J. W. Koo, R. C. Bagot, J. A. DiNieri, A. Nugent, E. Finkel, D. Chaudhury, R. Chandra, E. Riberio, J. Rabkin, E. Mouzon, R. Cachope, J. F. Cheer, M.-H. Han, D. M. Dietz, D. W. Self, Y. L. Hurd, V. Vialou, E. J. Nestler, $\Delta$ FosB Induction in Striatal Medium Spiny Neuron Subtypes in Response to Chronic Pharmacological, Emotional, and Optogenetic Stimuli. J. Neurosci. 33, 18381-18395 (2013).

58. A. Dumais, A. d. Lesage, M. Alda, G. Rouleau, M. Dumont, N. Chawky, M. Roy, J. j. Mann, C. Benkelfat, G. Turecki, Risk Factors for Suicide Completion in Major Depression: A Case-Control Study of Impulsive and Aggressive Behaviors in Men. AJP. 162, 2116-2124 (2005).

59. J. K. Mai, G. Paxinos, T. Voss, Atlas of the human brain (Elsevier, Acad. Press, Amsterdam, 3. ed., 2008).

60. M. Michino, P. Donthamsetti, T. Beuming, A. Banala, L. Duan, T. Roux, Y. Han, E. Trinquet, A. H. Newman, J. A. Javitch, L. Shi, A Single Glycine in Extracellular Loop 1 Is the Critical Determinant for Pharmacological Specificity of Dopamine D2 and D3 Receptors. Mol Pharmacol. 84, 854-864 (2013).

\section{Acknowledgments}

This work was supported by the Centre National de la Recherche Scientifique (CNRS); the Institut National de la Santé et de le Recherche Médicale National (INSERM) ; Sorbonne Université, Faculté des Sciences et Ingénierie ; Université de Bordeaux ; Institut National de Recherche pour l'Agriculture, l'Alimentation et l'Environnement (INRAE); Université Côte d'Azur ; the Agence Nationale pour la Recherche (ANR ; ANR-15-CE16-001 to P.V and P.T ; ANR-18-CE37-0003-02 to J.B and P.V ; ANR-10-IDEX-03-02 and ANR-16-CE16-0022 to P.T) ; the Fondation pour la Recherche Médicale (FRM ; DPA20140629798 to J.C ; DEQ20180339159 to J.B.) ; Institut de Recherche en Santé publique (IReSP) Aviesan APP-addiction 2019 (to P.V, P.T and J.B) ; NARSAD Young Investigator Grants from the Brain and Behavior Foundation (to P.T) ; the BioPsy labex excellence cluster (to J.C and P.V) ; the labex BRAIN (to P.T) and NIH grant MH54137 (to J.A.J.). The Douglas-Bell Canada Brain Bank is funded by platform support grants from the RQSHA and HBHL (CFREF). A.A and R.W. are recipients of PhD fellowship from the French Ministry of Research; A.P is the recipient of a $\mathrm{PhD}$ fellowship from the "Ecole Universitaire de Recherche" (EUR Neuro, Bordeaux Neurocampus); E.S.J is the recipient of a fourth-year PhD fellowship from the FRM. Authors would like to thank the imaging facility of the IBPS and the histology facility of the Institut du Cerveau et de Moëlle épinière (ICM).

\section{Authors contributions}

A.A performed most PLA, viral injections, behavioral studies, immunohistochemistry, confocal imaging, biochemistry and statistical analysis with the help of E.S.J; M.C.A; V.K; S.B, V.O and R.W. Dendritic spines analysis was performed by N.H and A.A. Electrophysiological recordings were performed by P.P 
and S.P.F. Viruses were designed by P.V and A.P.B and produced by C.J and A.P.B and V.S.P and A.P performed cAMP assays. G.T and N.M obtained, characterized and provided human brain samples. Y.Z and J.A.J. helped A.A for PLA experiments from human tissues and related quantifications. A.A; J.C; P.T; J.B and P.V designed experiments and wrote the manuscript, which was edited by the other authors.

\section{Competing interests}

The authors declare no competing interests 